\title{
Electro-acupuncture-induced neuroprotection is associated with activation of the IGF-1/PI3K/Akt pathway following adjacent dorsal root ganglionectomies in rats
}

\author{
TAO HU ${ }^{1,2 *}$, MIN-NAN LU ${ }^{3 *}$, BO CHEN $^{3 *}$, JUN TONG $^{4 *}$, RUI MAO $^{5 *}$, \\ SHAN-SHAN LI ${ }^{6}$, PIN DAI $^{1}$, YA-XIN TAN ${ }^{1}$ and YAN-BIN XIYANG ${ }^{1}$ \\ ${ }^{1}$ Institute of Neuroscience, Kunming Medical University, Kunming, Yunnan 650500; \\ ${ }^{2}$ Department of Laboratory Medicine, The Third People's Hospital of Yunnan Province, Kunming, Yunnan 650011; \\ ${ }^{3}$ Experiment Center for Medical Science Research; ${ }^{4}$ Physical Education Department; ${ }^{5}$ School of Stomatology; \\ ${ }^{6}$ Basic Medical College, Kunming Medical University, Kunming, Yunnan 650500, P.R. China
}

Received August 14, 2018; Accepted December 17, 2018

DOI: $10.3892 /$ ijmm.2018.4035

\begin{abstract}
The aim of the present study was to investigate the putative role and underlying mechanisms of insulin-like growth factor 1 (IGF-1) in mediating neuroplasticity in rats subjected to partial dorsal root ganglionectomies following electro-acupuncture (EA) treatment. The rats underwent bilateral removal of the L1-L4 and L6 dorsal root ganglia (DRG), sparing the L5 DRG, and were subsequently subjected to 28 days of EA treatment at two paired acupoints, zusanli (ST 36)-xuanzhong (GB 39) and futu (ST 32)-sanyinjiao (SP 6), as the EA Model group. Rats that received partial dorsal root ganglionectomies without EA treatment served as a control (Model group). Subsequently, herpes simplex virus (HSV)-IGF-1, HSV-small interfering (si) RNA-IGF-1 and the associated control vectors were injected into the L5 DRG of rats in the EA Model group. HSV-IGF-1 transfection enhanced EA-induced neuroplasticity, which manifested as partial recovery in locomotor function, remission hyperpathia, growth of DRG-derived spared fibers, increased expression of phosphorylated (p-) phosphatidylinositol 3-kinase (PI3K) and Akt, and increased pPI3K/PI3K and pAkt/Akt expression ratios. By contrast, HSV-siRNA-IGF-1 treatment attenuated these effects induced by HSV-IGF-1 transfection. The results additionally demonstrated that HSV-IGF-1 transfection augmented the outgrowth of neurites in cultured DRG neurons, and interference of the expression of IGF-1 retarded
\end{abstract}

Correspondence to: Dr Yan-Bin Xiyang, Institute of Neuroscience, Kunming Medical University, 1168 West Chunrong Road, Yuhua Avenue, Chenggong, Kunming, Yunnan 650500, P.R. China

E-mail: xiyang_neuro@126.com

*Contributed equally

Key words: insulin-like growth factor 1, electro-acupuncture, dorsal root ganglionectomy, phosphatidylinositol 3-kinase/Akt neurite outgrowth. Co-treatment with a PI3K inhibitor or Akt siRNA inhibited the aforementioned effects induced by the overexpression of IGF-1. In conclusion, the results of the present study demonstrated the crucial roles of IGF-1 in EA-induced neuroplasticity following adjacent dorsal root ganglionectomies in rats via the PI3K/Akt signaling pathway.

\section{Introduction}

The removal of afferents from adjacent dorsal root ganglia (DRG) may initiate the sprouting of novel sensory neurons to the denervated territory within the dorsal horn, which is known as neuroplasticity of the spinal cord. The compensatory sprouting of spared axons within the spinal cord following de-afferentation has been confirmed previously (1-4).

Acupuncture, a well-known tool of traditional Chinese medicine, has been reported to have therapeutic effects in patients suffering from spinal cord injury (SCI). Electro-acupuncture (EA), an effective acupuncture method, has been demonstrated to be a promising therapeutic method for functional recovery in patients with SCI (5). Previous studies have suggested that EA may promote functional repair (5-8) and enhance the intraspinal sprouting of spared afferents following SCI (9). Accumulating evidence suggested that alterations in the expression of neurotrophic factors (NTFs) may be associated with EA therapy-induced strengthening of spinal neuroplasticity following SCI $(10,11)$. However, the underlying mechanisms remain to be fully elucidated.

NTFs are important factors that influence neuronal plasticity following nerve injury (12). Insulin-like growth factors (IGFs), a sub-family of NTFs, are associated with neuronal growth. They maintain neuronal survival and promote the proliferation, differentiation and regeneration of injured neurons. In vitro and in vivo studies have demonstrated that the IGF system promotes the differentiation and proliferation of neurons and sustains their survival, thus being key in brain development (13-15). Insulin-like growth 
factor 1 (IGF-1), an isoform of the IGF family, is an NTF in the central nervous system (CNS) that functions via autocrine and paracrine signaling pathways (14). It has been demonstrated that IGF-1 exerts neuroprotective and proliferative effects through the promotion of cell survival (15), prevention of apoptosis (16) and stimulation of neurogenesis within the injured CNS (17). However, the underlying mechanisms are currently unclear.

Previous studies demonstrated that treadmill exercise promotes the recovery of motor function by suppressing apoptosis in the injured spinal cord. The beneficial effect of exercise may be attributed to an increase in expression of NTFs, including IGF-1, via activation of the phosphatidylinositol 3-kinase (PI3K)/Akt pathway (18). Impairment of insulin signaling induction by IGF-1 has been demonstrated to be involved in neurodegenerative diseases, including Alzheimer's disease (19-21) and Parkinson's disease (22-25). Our previous study investigated the effects of EA on the endogenous expression of IGF-1 in the spared DRG and the associated dorsal horns following adjacent dorsal root ganglionectomies in cats, in order to understand the role of IGF-1 in the EA-mediated promotion of spinal cord plasticity. The results demonstrated that the EA-induced increased expression of IGF-1 in the spared L6 DRG and the associated dorsal horns may be associated with the intraspinal sprouting of the DRG neurons in the cats subjected to adjacent dorsal root ganglionectomies (26). The aim of the present study was to investigate the putative role of the IGF-1/PI3K/Akt signaling pathway in the EA treatment induced-neuroplasticity of rats receiving partial dorsal root ganglionectomies.

\section{Materials and methods}

Ethical aspects. The use and care of animals were in accordance with animal care guidelines; they conformed to the Guide for the Care and Use of Laboratory Animals published by the US National Institutes of Health (Bethesda, MD, USA; publication no. 85-23, revised 1996) and the Care and Use Guidelines of Experimental Animals established by the Research Ethics Committee of Kunming University of China (Kunming, China; permit no. kmu-eac-2016047).

Construction of herpes simplex virus (HSV)-IGF-1 and $H S V$-small interfering (si)RNA-IGF-1 vectors. The HSV CL-1 sequence (Invitrogen; Thermo Fisher Scientific, Inc., Waltham, MA, USA), which does not contain ICP27, ICP4 or ICP34.5 genes, was prepared for the framework construction of the HSV vector. To generate the HSV-IGF-1 vector, cytomegalovirus (CMV), Woodchuck hepatitis virus post-transcriptional regulatory elements and poly $\mathrm{A}$ elements were ligated into a pNX plasmid (pNX-CMV; Takara Biotechnology, Co., Ltd., Dalian, China). Full-length open reading frame (ORF) cDNA clones of the IGF-1 gene (Rattus norvegicus; National Centre for Biotechnology Information gene ID: 24482), were purchased from Origene Technologies, Inc. (Rockville, MD, USA). A polymerase chain reaction (PCR) was conducted to amplify the cDNA clones of IGF-1 ORF concluding restriction sites for HindIII and XhoI enzymes, using the PCR Master Mix kit
(Invitrogen; Thermo Fisher Scientific, Inc.) for 30 cycles, consisting of denaturation at $94^{\circ} \mathrm{C}$ for $1 \mathrm{~min}$, annealing at $55^{\circ} \mathrm{C}$ for $30 \mathrm{sec}$, and extension at $72^{\circ} \mathrm{C}$ for $30 \mathrm{sec}$. The primers sequences were as follows: Sense, $5^{\prime}$-GACTGGACT TGCTATTGGGACC-3' and anti-sense 5'-TAGGCTATC TTGAGTCGGATT-3'. Subsequently, the ORF sequences were digested with HindIII and XhoI restriction enzymes, and ligated to pNX-CMV plasmid (pNX-CMV-IGF-1). The vector was subsequently sequenced by Sanger sequencing. For HSV-siRNA-IGF-1 construction, the recombinant pNX plasmid containing the U6 promoter and multiple cloning sites (pNX-U6) was ligated with siRNA-IGF-1 (pNX-siRNA-IGF-1). The transformants were screened and identified by PCR and restriction analyses. The pNX-CMV plasmid without cDNA clones of the IGF-1 gene ORF was used as blank control for pNX-CMV-IGF-1, and the pNX-U6 plasmid served as a control for siRNA-IGF-1. Subsequently, DNA of the framework construction of the HSV vector, pNX-CMV-IGF-1, pNX-CMV, pNX-siRNA-IGF-1 and pNX-U6 were transfected into the OG01 cell line (Takara Biotechnology, Co., Ltd.) by calcium phosphate coprecipitation. The OG01 cells were cultured in Dulbecco's modified Eagle's medium (DMEM) supplemented with $10 \%$ fetal bovine serum (FBS), $800 \mu \mathrm{g} / \mathrm{ml}$ neomycin and $700 \mu \mathrm{g} / \mathrm{ml}$ zeozin (all purchased from Invitrogen; Thermo Fisher Scientific, Inc.). The successfully transfected cell clones were selected for further PCR analysis, purification and hyperfiltration to obtain the target HSV vectors, HSV-IGF-1, HSV-pNX-CMV, HSV-siRNA-IGF-1 and HSV-pNX-U6, respectively. The HSV-pNX-CMV and HSV-pNX-U6 vectors served as blank controls for HSV-IGF-1 and HSV-siRNA-IGF-1, respectively.

Animal model. A total of 49 adult male Sprague-Dawley rats (weight 180-200 g, age 3-4 months), provided by the Laboratory Animal Center of Kunming Medical University, were used in the present study. The rats were housed with free access to food and water and exposed to a 12-h light/dark cycle in a restricted access, in a temperature-controlled (at $22^{\circ} \mathrm{C}$ ) animal centre with $55-60 \%$ humidity. All experimental procedures complied with the guidelines for the care and use of animals stipulated by the National Institutes of Health.

Details of the animal grouping are presented in Table I. Group I rats served as sham-operated controls that did not undergo DRG. The rats in Groups II-VII were subjected to surgical removal of the bilateral four lumbar (L1-L4) and sixth lumbar (L6) DRGs, sparing the L5 DRG. The rats in Group II received dorsal root ganglionectomy only. The rats in groups III, IV, V, VI and VII were subjected to bilateral dorsal root ganglionectomies followed immediately by electrical stimulation of the bilateral site at the zusanli (ST 36)-xuanzhong (GB 39) and futu (ST 32)-sanyinjiao (SP 6) acupoints (7). The rats in groups IV, V, VI and VII were injected with various HSV vectors at the site of the spared DRG. The rats were then subjected to further analysis. They were allowed to survive for 28 days post-operatively (dpo) as described in Table I.

Prior to the surgical procedure, the animals were deeply anesthetized with an intraperitoneal injection of pentobarbital sodium solution $(2 \% ; 40 \mathrm{mg} / \mathrm{kg}$ body weight). A 
Table I. Animal grouping and treatments.

\begin{tabular}{lcll}
\hline Group & Rats (n) & \multicolumn{1}{c}{ Treatment } & Experimental methods \\
\hline I & 7 & Sham-operated & RT-qPCR/WB/IHC/BBB/MWT/TWL \\
II & 7 & Model (operation only) & RT-qPCR/WB/IHC/BBB/MWT/TWL \\
III & 7 & EA Model (operation + EA) & RT-qPCR/WB/IHC/BBB/MWT/TWL \\
IV & 7 & EA Model + HSV-IGF-1 & RT-qPCR/WB/IHC/BBB/MWT/TWL \\
V & 7 & EA Model + HSV-pNX-CMV & RT-qPCR/WB/IHC/BBB/MWT/TWL \\
VI & 7 & EA Model + HSV-siRNA-IGF-1 & RT-qPCR/WB/IHC/BBB/MWT/TWL \\
VII & 7 & EA Model + HSV-pNX-U6 & RT-qPCR/WB/IHC/BBB/MWT/TWL \\
\hline
\end{tabular}

Model, dorsal root ganglionectomy injury; EA, electro-acupuncture; IGF-1, insulin-like growth factor 1; siRNA, small interfering RNA; RT-qPCR, reverse transcription-quantitative polymerase chain reaction; WB, western blotting; IHC, immunohistochemistry; BBB, Basso, Beattie, Bresnahan score; MWT, mechanical withdrawal threshold; TWL, thermal withdrawal latency.

bilateral hemilaminectomy was performed at lumbosacral levels as described previously (7). The articular processes of the respective vertebrae were removed with a pair of rongeurs and the dura was cut with a small pair of scissors. The L1-L4 and L6 DRGs were exposed and removed at their respective intervertebral foramina, leaving the L5 DRG intact. Following surgery, the overlaying skin and muscle were sutured, and suitable post-operative care was provided regularly.

All injections into the DRG were performed using a microprocessor-controlled injection system employing direct piston displacement (Nanoliter 2000; World Precision Instruments, Sarasota, FL, USA) mounted on a micromanipulator as described previously (27). For direct injection into the spared L5 DRG, the foramen was enlarged in a cephalad direction using the rongeurs, in order to remove a 1-mm-deep crescent of laminar bone, exposing the distal third of the DRG. A total of $2 \mu \mathrm{l}$ HSV-IGF-1, HSV-siRNA-IGF-1, or the associated control HSV-pNX-CMV and HSV-pNX-U6 vectors, respectively, were injected into the DRG at a rate of $20 \mathrm{nl} / \mathrm{sec}$. Following completion of the injection, the pipette was left in place for $5 \mathrm{~min}$ prior to removal to allow the fluid to distribute and the pressure within the tissue to equalize. The wound was closed in layers.

EA treatment. The rats in Groups III-VII were subjected to EA treatment at two paired acupoints, zusanli (ST 36)-xuanzhong (GB 39) and futu (ST 32)-sanyinjiao (SP 6) $(7,28)$. Their locations were as follows: ST 36 is located $0.5 \mathrm{~cm}$ below the front of the fibula head; GB 39, $1.0 \mathrm{~cm}$ above the front of the lateral malleolus; ST 32, $1.5 \mathrm{~cm}$ above the lower end of the patella; and SP $6,0.5 \mathrm{~cm}$ above the posterior end of the medial malleolus. These acupoints are known to lie in the dermatome of L5 as described in previous report (7). The rats were placed in plastic holders to prevent movement of their body and hindlimbs when treated with EA, with the animals remaining awake without anesthesia in a convenient and safe manner. When the rats had been acclimated to the holders, EA stimulation was performed. During EA treatment, the conditions of the rats were monitored. If any signs of discomfort were observed in the rat induced by EA, the EA treatment was immediately terminated. Every day, these acupoints were stimulated alternately at a frequency of
98-HZ pulses/min at $5 \mathrm{~V}(98 \mathrm{HZ} / 5 \mathrm{~V})$, at $1.0 \mathrm{~mA}$ for $30 \mathrm{~min}$ delivered by an EA apparatus (Hans electro-stimulator; Nanjing Jisheng Medical Technology Co., Ltd., Nanjing, China) with the electrodes connected to two acupuncture needles. The electrodes were replaced every 15 min during each acupuncture. A preliminary experiment was performed to evaluate the effects of different stimulation intensities of EA on rats and select the optimal intensity and duration. An EA intensity at $98 \mathrm{HZ} / 5 \mathrm{~V}$ was selected for the present study (data not shown).

Evaluation of hindlimb locomotor function. The hindlimb locomotor function of the rats in each group was assessed using the Basso, Beattie, Bresnahan (BBB) rating scale (29) at 7, 14, 21 and 28 dpo. The BBB scores following transfection ranged between 0 and 21 . The animals were allowed to walk around freely in an open field for $4 \mathrm{~min}$, during which hindlimb movements were closely observed. Three double-blinded individuals conducted the evaluations, and their average scores were calculated. All behavioral evaluations were performed daily at 8:00-9:00 a.m. following evacuation of the bladder.

Mechanical withdrawal threshold (MWT) test. MWT was determined for each hind-paw using von Frey filaments (0.4-15.0 g; Stoelting, Co., Wood Dale, IL, USA) and an 'up and down' procedure between 10:00 a.m. and 12:00 p.m. every day post-surgery, as previously described (30-34). If a withdrawal response to a particular hair was observed at least five times, the value of that hair in grams was considered as the withdrawal threshold. If a withdrawal response did not occur with the $15.0 \mathrm{~g}$ von Frey filament, it was considered a painless response. Paw withdrawal due to animal movement was not considered a positive response. The data were analyzed using the Dixon non-parametric test $(31,35)$. Details of the treatment groups are presented in Table I. A total of seven animals were included in each treatment group.

Thermal withdrawal latency (TWL) detection. TWL was assessed to determine the thermal sensitivity of rats using a Hargreave's heat source (3A) with a Halogen Photo Optic lamp 
$(15 \mathrm{~V}, 150 \mathrm{~W})(36)$. The average temperature at the animal's hind-paw surface was $36.2^{\circ} \mathrm{C}$ at $10 \mathrm{sec}, 39.2^{\circ} \mathrm{C}$ at $14 \mathrm{sec}$ and $41.3^{\circ} \mathrm{C}$ at $16 \mathrm{sec}$. The animals were placed in a clear Plexiglass box on an elevated platform and allowed to acclimatize for $10 \mathrm{~min}$. A radiant heat source with constant intensity was aimed at the mid-planter area of the hind-paw.

The paw TWL was recorded using a timer three times with 10-min intervals between each trial, and the mean of these three trials was then calculated. A cut-off time of $30 \mathrm{sec}$ was used to prevent potential tissue damage. If no paw withdrawal occurred by $30 \mathrm{sec}$, the radiant heat was removed and TWL was recorded as $30 \mathrm{sec}(34)$.

$D R G$ culture. DRGs were isolated from the neonatal Sprague-Dawley rats as described previously (37) with minor modifications. The rats were sacrificed by exposure to increasing concentrations of $\mathrm{CO}_{2}$ followed by cervical dislocation. Ganglia from all spinal levels were dissected in chilled PBS (Invitrogen; Thermo Fisher Scientific, Inc.). The DRG tissues were dissociated following enzymatic digestion in $0.25 \%$ trypsin-EDTA (Gibco; Thermo Fisher Scientific, Inc.). The tissue was triturated using a 1-ml Gilson pipette. The DRG neurons were separated from myelin and cellular debris by centrifugation for $10 \mathrm{~min}$ at $145 \mathrm{x} \mathrm{g}$ and $20-25^{\circ} \mathrm{C}$ in $2 \mathrm{ml}$ DMEM/Ham's F12 (F12; Gibco; Thermo Fisher Scientific, Inc.) supplemented with $10 \%$ FBS. Pellets containing the DRG neurons were resuspended in DMEM/F12 media, containing $100 \mathrm{U} / \mathrm{ml}$ penicillin $\mathrm{G}, 100 \mu \mathrm{g} / \mathrm{ml}$ streptomycin (Gibco; Thermo Fisher Scientific, Inc.) and 10\% FBS (Invitrogen; Thermo Fisher Scientific, Inc.). The cells were maintained at $37^{\circ} \mathrm{C}, 95 \% \mathrm{O}_{2}$ and $5 \% \mathrm{CO}_{2}$ prior to analysis.

DRG neuron transfection. The HSV-IGF-1, HSV-siRNA-IGF-1, HSV-pNX-CMV and HSV-pNX-U6 vectors were each transfected into DRG neurons using Lipofectamine ${ }^{\circledR} 2000$ (Invitrogen; Thermo Fisher Scientific, Inc.) according to the manufacturer's protocol. In total, $\sim 1 \times 10^{5}$ DRG neurons were seeded in $35-\mathrm{mm}$ plates (24-well) and maintained in a conditioned medium consisting of DMEM/F12 (1:1) supplemented with $100 \mathrm{U} / \mathrm{ml}$ penicillin $\mathrm{G}, 100 \mu \mathrm{g} / \mathrm{ml}$ streptomycin (Gibco; Thermo Fisher Scientific, Inc.) and 10\% FBS. The cells were incubated at $37^{\circ} \mathrm{C}, 5 \% \mathrm{CO}_{2}$ overnight and the fresh DMEM/F12 medium was replaced every 2 days. When the cultured cells reached $60 \%$ confluence, they were transfected with the HSV-IGF-1, HSV-siRNA-IGF-1, HSV-pNX-CMV or HSV-pNX-U6 plasmids. At 3 days post-transfection, the cells were digested with $0.25 \%$ trypsin for $8 \mathrm{~min}$, followed by the addition of FBS containing medium to inhibit trypsin. The samples were subsequently centrifuged at $500 \mathrm{xg}$ and $4^{\circ} \mathrm{C}$ for 30 min. To confirm successful transfection of the HSV-IGF-1 vector constructs, the cells were subsequently subjected to reverse transcription-quantitative PCR (RT-qPCR) and western blot analyses. The effects of HSV-IGF-1 and HSV-siRNA-IGF-1 on DRG neural growth were additionally evaluated by measuring the axon length, areas of neurons and cell numbers using a LEICA DMI6000B microscope (LAS AF system; Leica Microsystems GmbH, Wetzlar, Germany; magnification, x200).

The PI3K/Akt inhibitor and Akt siRNA were used to investigate the role of the PI3K/Akt signaling pathway in neuroprotection induced by IGF-1. A specific synthetic inhibitor of PI3K, LY294002 (cat. no. sc391584; Santa Cruz Biotechnology, Inc., Dallas, TX, USA) was used to pre-treat the DRG neurons for $30 \mathrm{~min}$ at a final concentration of $20 \mu \mathrm{mol} / 1$ (38). The cells were subsequently transfected with HSV-IGF-1 using the aforementioned procedures. The cells in the control group were treated with dimethyl sulfoxide.

Specific siRNA duplexes targeting Akt were used to inhibit the expression of Akt. The siRNA sequences were as follows: 5'-UUCAGGUACUCAAACUCGUUCAUGG-3' and 5'-CCAUGAACGAGUUUGAGUACCUGAA-3' (39), and were synthesized by Invitrogen; Thermo Fisher Scientific, Inc. Scrambled siRNA duplexes served as a control. The Akt or scrambled siRNAs were transfected into DRG neurons using Lipofectamine ${ }^{\circledR} 2000$ (Invitrogen; Thermo Fisher Scientific, Inc.) following HSV-IGF-1 transfection, according to the manufacturer's protocol.

$R T-q P C R$ analysis. RT-qPCR was used to quantify the mRNA expression levels of IGF-1. All the primers employed in the present study were synthesized by Invitrogen; Thermo Fisher Scientific, Inc. The cultured DRG neurons and spared L5 DRG samples in the rats were additionally obtained for IGF-1 detection, as previously described (40). TRIzol ${ }^{\circledR}$ reagent (Invitrogen; Thermo Fisher Scientific, Inc.) was used to extract the total RNA, according to the manufacturer's protocol. Total RNA was eluted in $20 \mu 1$ RNase-free DNase I to remove DNA contamination. RNA samples were kept on ice and their concentrations were measured using a NanoDrop spectrophotometer (NanoDrop-1000; NanoDrop Technologies; Thermo Fisher Scientific, Inc., Wilmington, De, USA). For RNA amplification, the first-strand cDNA was synthesized from $2 \mu \mathrm{g}$ total RNA, using Revert Aid ${ }^{\mathrm{TM}}$ First Strand cDNA Synthesis kit (Takara Biotechnology Co., Ltd.). In total, $2 \mu \mathrm{g}$ total RNA was incubated with $1 \mu \mathrm{l}$ oligo (dT) 18 primers and $10 \mu \mathrm{l}$ diethylpyrocarbonate-treated water at $70^{\circ} \mathrm{C}$ for $5 \mathrm{~min}$, followed by cooling on ice. This mixture was subsequently supplied with $4 \mu 15 \mathrm{X}$ reaction buffer, $1 \mu$ l Riboblock ${ }^{\mathrm{TM}}$ Ribonuclease inhibitor and $10 \mathrm{mM}$ dNTP, and incubated at $37^{\circ} \mathrm{C}$ for $5 \mathrm{~min}$. For cDNA synthesis, $1 \mu 1$ Revert Aid ${ }^{\mathrm{TM}}$ M-Mulv Reverse Transcriptase was added to this mixture for a final incubation at $42^{\circ} \mathrm{C}$ for $1 \mathrm{~h}$, which was terminated by heating at $70^{\circ} \mathrm{C}$ for $10 \mathrm{~min}$. PCR was subsequently conducted using the $2 \mathrm{x}$ Mix SYBR-Green I (Beijing Biosea Biotechnology Co., Ltd., Beijing, China; $10 \mu \mathrm{l})$, primer $(0.25 \mu \mathrm{l} ; 10 \mathrm{pmol} / \mathrm{l})$, template DNA $(1 \mu \mathrm{l})$ and sterile water $(8.5 \mu \mathrm{l})$. qPCR amplification was performed at $95^{\circ} \mathrm{C}$ for $3 \mathrm{~min}$, followed by 39 thermocycling steps consisting of denaturation at $94^{\circ} \mathrm{C}$ for $30 \mathrm{sec}$, annealing at $58^{\circ} \mathrm{C}$ for $30 \mathrm{sec}$, and extension at $72^{\circ} \mathrm{C}$ for $30 \mathrm{sec}$. The primer sequences were as follows: GAPDH forward, 5'-CGA GATCCCTCCAAAATCAA-3' and reverse, 5'-TTCACACCC ATGACGAACAT-3'; and IGF-1 forward, 5'-GGTGGATGC TCTTCAGTTC-3' and reverse, 5'-TTTGTAGGCTTCAGT GGG-3'. The relative quantification cycle $(\mathrm{Cq})$ method was used to compare target gene expression between samples. Fold changes in target gene expression were determined relative to a blank control following normalization to the GAPDH housekeeping gene using the $2^{-\Delta \Delta \mathrm{Cq}}$ method (41).

Western blot analysis. Western blot analysis was performed as previously described $(42,43)$. The cultured DRG neurons and 
spared L5 DRG in rats were harvested and separately homogenized on ice in $10 \mathrm{mM}$ Tris- $\mathrm{HCl}$ buffer ( $\mathrm{pH} 7.4), 10 \mathrm{mM}$ EDTA, 30\% Triton-1000, 10\% SDS, a protease inhibitor cocktail (Roche Diagnostics, Indianapolis, IN, USA) and $\mathrm{NaCl}$ using a homogenizer. The homogenates were centrifuged at $12,000 \times \mathrm{g}$ for $30 \mathrm{~min}$ at $4^{\circ} \mathrm{C}$. The supernatant was collected and the protein concentration was assayed with bicinchoninic acid reagent (Sigma-Aldrich; Merck KGaA, Darmstadt, Germany) according to the manufacturer's protocol. A total of $50 \mu \mathrm{g}$ protein was used for 10\% SDS-PAGE (Sigma-Aldrich; Merck KGaA). The protein was subsequently transferred to a nitrocellulose membrane (Sigma-Aldrich; Merck KGaA), which was incubated in TBS and Tween-20 containing $5 \%$ non-fat milk powder (both Invitrogen; Thermo Fisher Scientific, Inc.) at $4^{\circ} \mathrm{C}$ for $8 \mathrm{~h}$ to reduce the nonspecific reaction. The membrane was subsequently incubated with primary antibodies at $4^{\circ} \mathrm{C}$ for $12 \mathrm{~h}$. $\beta$-actin (mouse monoclonal anti- $\beta$-actin; cat. no. sc-69879; dilution, 1:800; Santa Cruz Biotechnology, Inc.) was used as a reference and subtracted for net alterations in expression. Goat polyclonal anti-IGF-1 antibody (cat. no. ab106836; dilution, 1:1,000; Abcam, Cambridge, UK), mouse monoclonal anti-PI3K antibody (cat. no. 13666; dilution, 1:1,000), rabbit anti-phosphorylated (p)-PI3K antibody (cat. no. 4228; dilution, 1:800), and rabbit polyclonal anti-p-Akt (cat. no. 9275; dilution, 1:1,000) and anti-Akt antibodies (cat. no. 9272; dilution, 1:1,000; all Cell Signaling Technology, Inc., Danvers, MA, USA), were used as the primary antibodies. Horseradish peroxidase-conjugated anti-goat antibody for IGF-1 (cat. no. PI-9500; dilution, 1:500), horseradish peroxidase-conjugated anti-mouse antibody for PI3K (cat. no. PI-2000; dilution, 1:3,000) and horseradish peroxidase-conjugated anti-rabbit antibody for p-Akt and Akt (cat. no. PI-1000; dilution, 1:2,500; all Vector Laboratories, Inc., Burlingame, CA, USA) were used as the secondary antibodies. The membranes were subsequently incubated in the matched secondary antibodies at $20-25^{\circ} \mathrm{C}$ for $2 \mathrm{~h}$. Enhanced chemiluminescence luminol reagent (Beyotime Institute of Biotechnology, Shanghai, China) was used for protein quantity determination. The densitometric analysis of the target protein bands were analyzed using Bio-Rad Gel Imagining system (ChemiDoc $^{\mathrm{TM}}$ XRS+) with Quantity One software v4.6.6 (all Bio-Rad Laboratories, Inc., Hercules, CA, USA) for each group in order to quantity the protein expression levels.

Immunohistochemistry (IHC) detection. The L4 and L5 spinal cord segments of the rats were collected and post-fixed for $24 \mathrm{~h}$ using 4\% paraformaldehyde (Sigma-Aldrich; Merck $\mathrm{KGaA})$ at $4^{\circ} \mathrm{C}$ for IHC detection. The spinal cord tissue sections $(20-\mu \mathrm{m}$ thickness) were processed as described previously $(9,10)$. The sections were incubated with rabbit anti-rat polyclonal antibodies, calcitonin gene-related peptide (CGRP; cat. nos. 57053; dilution, 1:500, Santa Cruz Biotechnology, Inc.) and grow th-associated protein (GAP)43 (cat. no. ab12274; dilution, 1:1,000; Abcam, Cambridge, MA, USA), at $4^{\circ} \mathrm{C}$ overnight. Negative controls were incubated in 2\% goat serum (Santa Cruz Biotechnology, Inc.) instead of the primary antibody. Finally, the sections were detected by diaminobenzidine staining, according to the manufacturer's protocol (DAB color development kit; cat. no. P0203; Beyotime Institute of Biotechnology). In total,
$0.5 \mathrm{ml}$ DAB staining solution $\mathrm{A}, 0.5 \mathrm{ml} \mathrm{DAB}$ staining solution $\mathrm{B}$ and $1 \mathrm{ml}$ DAB staining working solution were prepared and gently mixed. The sections were incubated with the mixture at $20-25^{\circ} \mathrm{C}$ for $5 \mathrm{~min}$. The stain reaction was stopped by adding sterile water to wipe off the staining solutions. Subsequently, the sections were dried at $45^{\circ} \mathrm{C}$ for $2 \mathrm{~h}$, followed by soaking in $75 \%$ ethyl alcohol for $10 \mathrm{~min}$, $80 \%$ ethyl alcohol for $10 \mathrm{~min}, 85 \%$ ethyl alcohol for $10 \mathrm{~min}$, $90 \%$ ethyl alcohol for $10 \mathrm{~min}, 95 \%$ ethyl alcohol for $10 \mathrm{~min}$, absolute ethyl alcohol for $10 \mathrm{~min}$ and $100 \%$ xylene for $20 \mathrm{~min}$ at $20-25^{\circ} \mathrm{C}$. The sections were covered with a clean coverslip. The immunoreactive staining was observed under a light microscope (Leica Microsystems $\mathrm{GmbH}$; magnification, $\mathrm{x} 200$ or 400).

Statistical analysis. Each experiment was repeated three times. One-way analysis of variance or the Student-Newman-Keuls and the least significant difference or Dunnett's T3 post hoc tests were used to infer significant differences in the data. SPSS 13.5 covariance software for Windows (SPSS, Inc., Chicago, IL, USA) was used to perform these analyses. $\mathrm{P}<0.05$ was considered to indicate a statistically significant difference. All values are expressed as the mean \pm standard deviation.

\section{Results}

Effects of HSV on the expression of IGF-1. The expression of IGF-1 at the mRNA and protein levels were detected in all experimental groups. Bilateral dorsal root ganglionectomies significantly decreased the levels of IGF-1 (sham-operated group vs. Model group; $\mathrm{P}<0.05)$, whereas EA treatment partially rescued the expression of IGF-1 (Model group vs. EA Model group; $\mathrm{P}<0.05$; Fig. 1A).

Following HSV-IGF-1 treatment, the expression of IGF-1 increased 1.6-fold in the spared L5 DRG of the HSV-IGF-1-injected EA Model group (HSV-IGF-1 vs. HSV-pNX-CMV; P<0.05; Fig. 1A). By contrast, HSV-siRNA-IGF-1 injection significantly reduced the expression of IGF-1 in the spared L5 DRG (HSV-siRNA-I GF-1 vs. HSV-pNX-U6; P<0.05; Fig. 1B).

In the cultured DRGs, treatment with HSV-IGF-1 also increased the levels of IGF-1 (HSV-IGF-1 vs. HSV-pNX-CMV; $\mathrm{P}<0.05$; Fig. 1C), whereas injection with HSV-siRNA-IGF-1 significantly decreased the expression of IGF-1 (HSV-siRNA-IGF-1 vs. HSV-pNX-U6; P<0.05; Fig. 1C). These results demonstrated that either HSV-IGF-1 or HSV-siRNA-IGF-1 treatment effectively resulted in the upregulation or downregulation of IGF-1 in the cultured DRGs, respectively.

Locomotor function evaluation. The results demonstrated that the baseline BBB score of the sham-operated rats was equal to 21 . The BBB scores decreased in the injury model group following bilateral dorsal root ganglionectomies, and then gradually increased between 7 and 28 dpo. In addition, the BBB scores of the EA group were significantly elevated between 14 and 28 dpo compared with those of the injured group (EA model vs. Model; $\mathrm{P}<0.05$ ). These data suggest that EA effectively promotes functional locomotor recovery in the hindlimbs (Fig. 2A). 
A

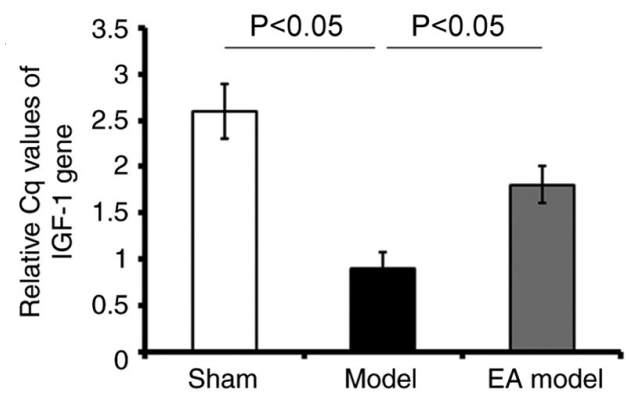

$\mathrm{B}$

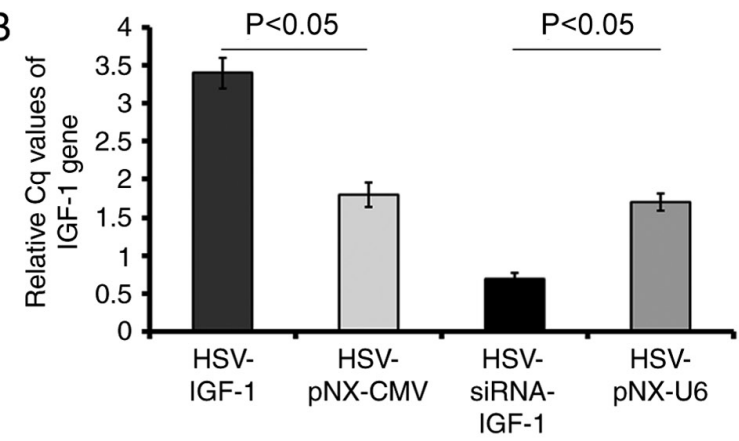

$\mathrm{C}$

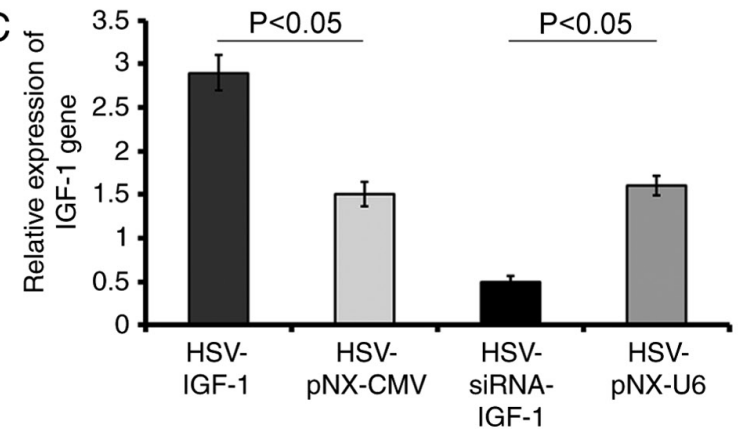

$\longrightarrow-\beta$-actin
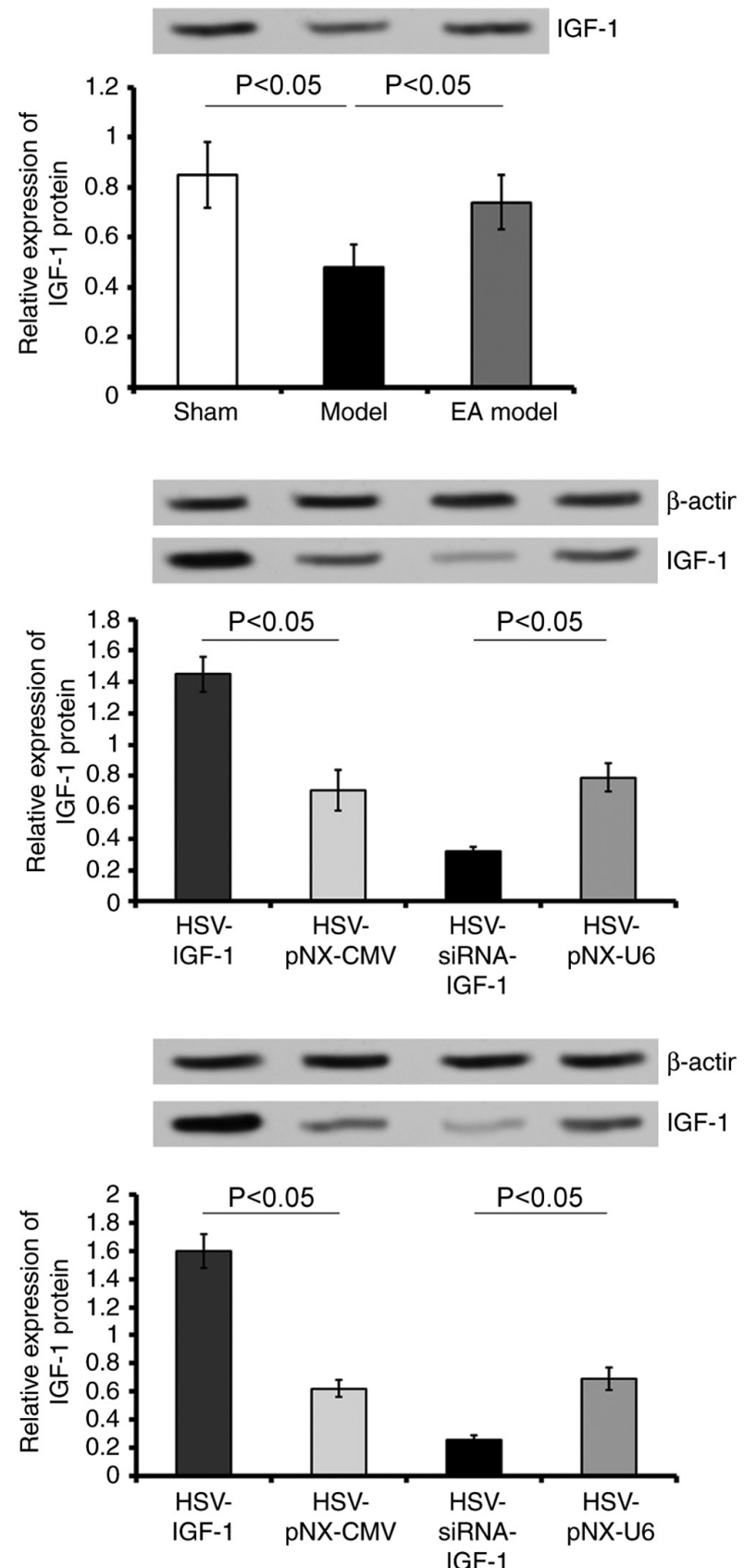

Figure 1. Evaluation of the expression of IGF-1 in vivo and in vitro. (A) Gene and protein levels of IGF-1 were detected in the L5 spared DRG of rats in the Sham, Model, and EA Model groups. (B) Gene and protein levels of IGF-1 were detected in EA model rats injected with artificially synthetic HSV-IGF-1, HSV-siRNA-IGF-1, HSV-pNX-CMV or HSV-pNX-U6 into the spared L5 DRG. (C) Gene and protein levels of IGF-1 were detected in cultured DRGs transfected with HSV-IGF-1, HSV-siRNA-IGF-1, HSV-pNX-CMV or HSV-pNX-U6. Values are plotted as the mean \pm standard deviation (n=7). Sham, sham-operated; Model, bilateral dorsal root ganglionectomy injury; EA, electro-acupuncture treatment post-injury; DRG, dorsal root ganglia; IGF-1, insulin-like growth factor 1; siRNA, small interfering RNA.

In the dorsal root ganglionectomy groups of rats treated with EA (EA Model), the overexpression of IGF-1 by HSV-IGF-1 injection in the spared L5 DRG significantly increased the BBB scores between 14 and 28 dpo compared with those in the blank vector-treated rats (HSV-IGF-1 vs. HSV-pNX-CMV; P<0.05; Fig. 2B). By contrast, interference of the endogenous expression of IGF-1 by HSV-siRNA-IGF-1 injection induced a significant decrease in the BBB scores of the EA Model rats (HSV-siRNA-IGF-1 vs. HSV-pNX-U6; $\mathrm{P}<0.05$; Fig. 2B). The results suggest that the overexpression of IGF-1 enhanced EA-induced neuroprotection following the dorsal root ganglionectomy procedure, whereas the inhibition of IGF-1 partially neutralized EA-induced locomotor functional recovery in the hindlimbs.

MWT and TWL tests. In the sham-operated rats, there were no significant changes in the MWT or TWL during the entire experimental period. The MWT was significantly decreased in the bilateral dorsal root ganglionectomy group (Model vs. sham, $\mathrm{P}<0.05$ ), and was partially recovered in the EA Model between 

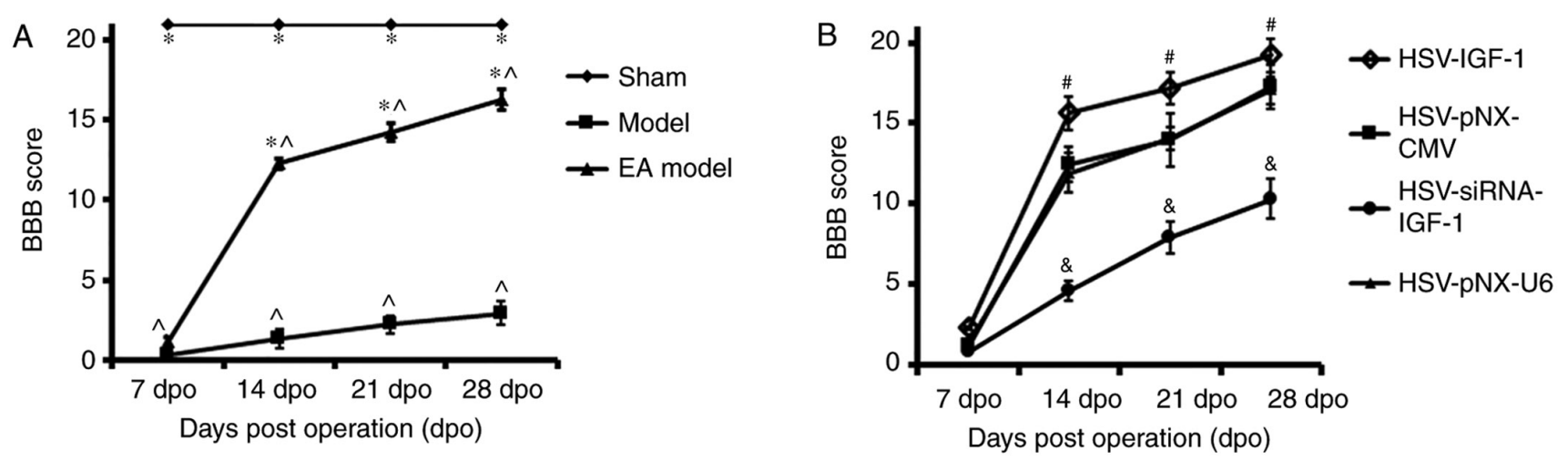

Figure 2. Evaluation of locomotor function in different groups. (A) BBB score evaluation was performed at 7, 14, 21 and 28 dpo, in the Sham, Model, and EA Model rats. "P<0.05 vs. Model; ${ }^{\wedge} \mathrm{P}<0.05$ vs. Sham. (B) BBB score evaluation in the EA Model injected with artificially synthetic HSV-IGF-1, HSV-siRNA-IGF-1, HSV-pNX-CMV or HSV-pNX-U6 into the spared L5 DRG. " P<0.05 vs. HSV-pNX-CMV; ${ }^{\circledR} \mathrm{P}<0.05$ vs. HSV-pNX-U6. Values are plotted as the mean \pm standard deviation $(\mathrm{n}=7)$. Sham, sham-operated; Model, bilateral dorsal root ganglionectomy injury; EA, electro-acupuncture treatment post-injury; BBB, Basso, Beattie, Bresnahan; IGF-1, insulin-like growth factor 1; siRNA, small interfering RNA.
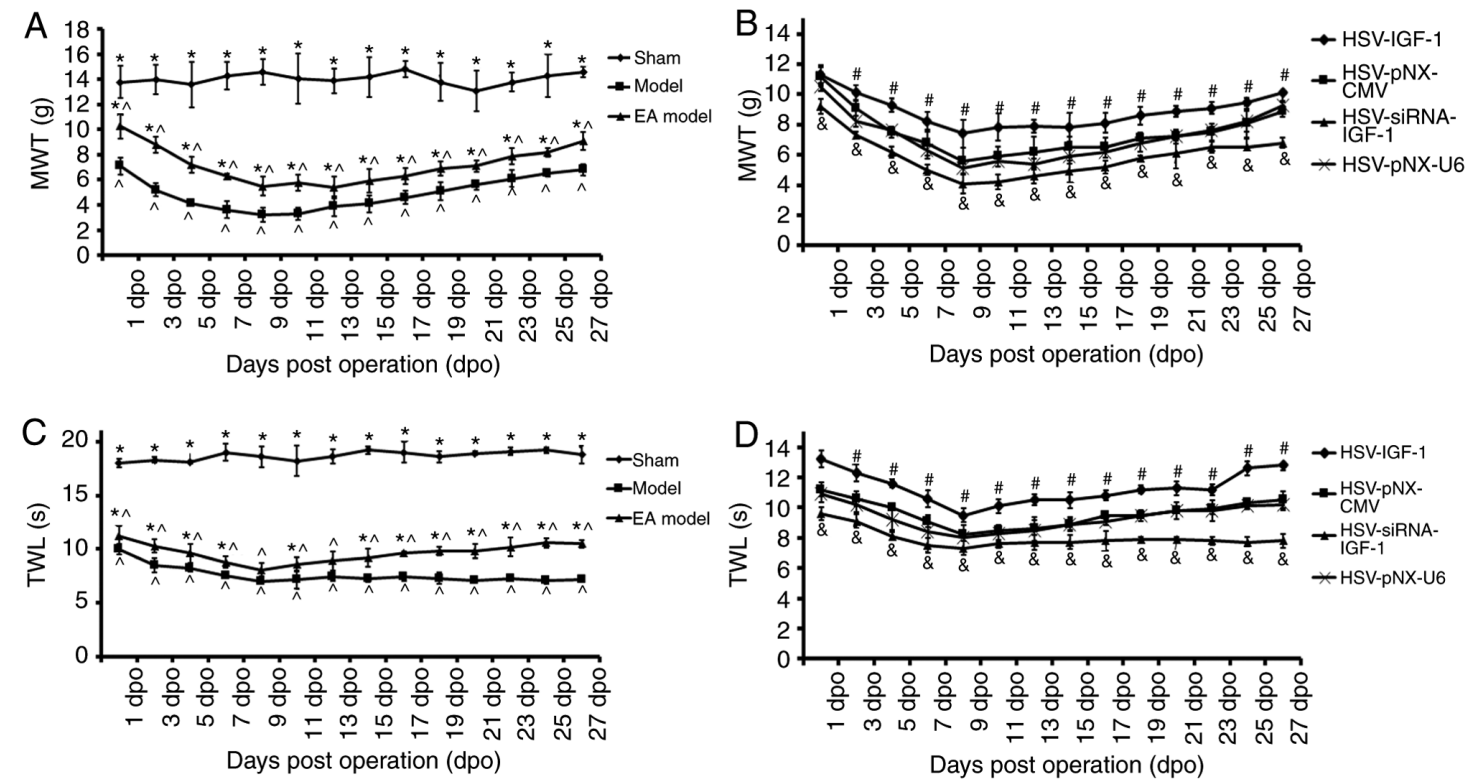

Figure 3. Effects of IGF-1 on neuropathic pain of rats with dorsal root ganglionectomies treated with EA. (A) MWT evaluation in rats in the Sham, Model, and EA Model rats. "P $<0.05$ vs. Model; ${ }^{\wedge} \mathrm{P}<0.05$ vs. Sham. (B) MWT evaluation in the EA model rats injected with artificial HSV-IGF-1, HSV-siRNA-IGF-1, HSV-pNX-CMV or HSV-pNX-U6 into the spared L5 DRG. ${ }^{\text {P }}<0.05$ vs. HSV-pNX-CMV, ${ }^{\&} \mathrm{P}<0.05$ vs. HSV-pNX-U6, $\mathrm{P}<0.05$. (C) TWL test in rats in the sham, Model, and EA model rats. "P<0.05 vs. Model; ${ }^{\wedge} \mathrm{P}<0.05$ vs. Sham. (D) TWL test in the EA model rats injected with artificial HSV-IGF-1, HSV-siRNA-IGF-1, HSV-pNX-CMV or HSV-pNX-U6 into the spared L5 DRG. " P<0.05 vs. HSV-pNX-CMV; ${ }^{\circledR} \mathrm{P}<0.05$ vs. HSV-pNX-U6. Values are plotted as the mean \pm standard deviation ( $\mathrm{n}=7$ ). Sham, sham-operated; Model, bilateral dorsal root ganglionectomy injury; EA Model, electro-acupuncture treatment post-injury; MWT, mechanical withdrawal threshold; TWL, thermal withdrawal latency; IGF-1, insulin-like growth factor 1; siRNA, small interfering RNA.

1 and 27 dpo (EA Model vs. Model; P<0.05; Fig. 3A). The MWT of the Model group reached its lowest threshold at 9 dpo, and this decrease was maintained until 13 dpo (Fig. 3A). HSV-IGF-1 injection induced partial recovery of the MWT in the EA Model group (HSV-IGF-1 vs. HSV-pNX-CMV, P<0.05). By contrast, MWT showed a further decrease and reached its lowest threshold at 9 dpo in the HSV-siRNA-IGF-1-treated rats (HSV-siRNA-IGF-1 vs. HSV-pNX-U6; P<0.05; Fig. 3B). No significant differences were observed between the HSV-pNX-U6 and HSV-pNX-CMV-injected EA Model groups. Similar alterations in TWL in each experimental group were observed (Fig. 3C and D). These results suggested that manipulating the expression of endogenous IGF-1 had significant effects on neuropathic pain following deafferentation injury.
IGF-1 increases CGRP- and GAP-43-immunopositive reactions (IRs) in the EA Model group via the regulation of PI3K/Akt. CGRP-positive fibers were observed in the lamina I-V and motor neurons of spinal ventral horns in the sham-operated and Model group (Fig. 4), respectively. CGRP-IRs were observed in the spinal cords of rats in the EA Model group (Fig. 4). HSV-IGF-1 treatment enhanced CGRP-immunopositive staining, compared with that in the HSV-pNX-CMV-treated group (Fig. 4). However, HSV-siRNA-IGF-1 treatment was associated with reduced CGRP-immunopositive staining (HSV-siRNA-IGF-1 vs. HSV-pNX-U6; Fig. 4).

GAP-43-immunopositive staining was additionally enhanced in the EA Model group relative to that in the 


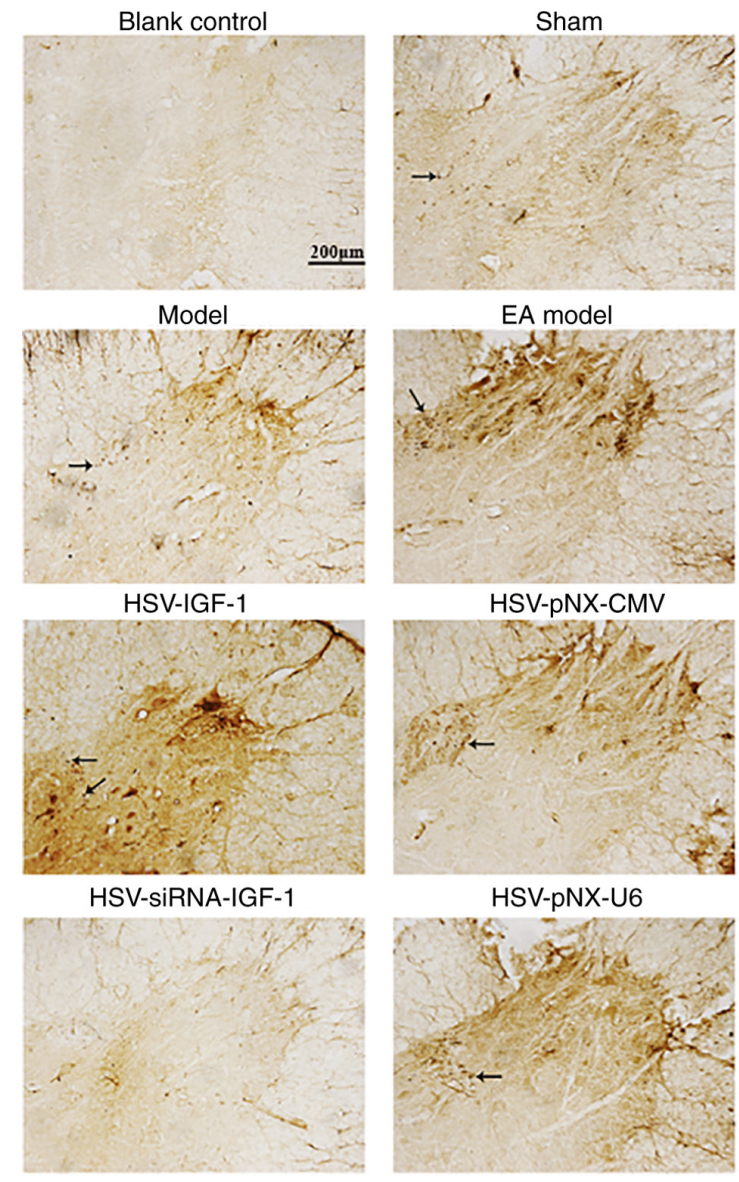

Figure 4. CGRP-positive fibers in different groups. Arrows indicate the representative files of CGRP-stained files. Phosphate-buffered saline was substituted for the anti-CGRP antibody in the blank control ones. Magnification, x200. Sham, sham-operated; Model, bilateral dorsal root ganglionectomy injury; EA Model, electro-acupuncture treatment post-injury; CGRP, calcitonin gene-related peptide; IGF-1, insulin-like growth factor 1 ; siRNA, small interfering RNA.

sham-operated group and injured Model group (Fig. 5). GAP-43-IRs were also increased in the HSV-IGF-1-treated group and reduced in the HSV-siRNA-IGF-1-treated group, compared with their respective control groups, HSV-pNX-CMV and HSV-pNX-U6, respectively (Fig. 5).

Similarly, the expression levels of pPI3K and pAkt were reduced following bilateral dorsal root ganglionectomy (sham-operated group vs. Model group, $\mathrm{P}<0.05$; Fig. 6). HSV-IGF-1 injection enhanced the expression of PI3K and pAkt and the ratio of pAkt/Akt in the EA Model rats (Fig. 6A and B, $\mathrm{P}<0.05)$. However, treatment with HSV-siRNA-IGF-1 reduced the ratios of $\mathrm{p}-\mathrm{PI} 3 \mathrm{~K} / \mathrm{PI} 3 \mathrm{~K}$ and $\mathrm{pAkt} / \mathrm{Akt}$ compared with those in the HSV-pNX-U6-treated group $(\mathrm{P}<0.05$; Fig. 6A and $\mathrm{B})$.

Overexpression of IGF-1 induces DRG neuronal growth by activating PI3K/Akt. Treatment with HSV-IGF-1 increased the number and extension of DRG neurons, compared with the HSV-pNX-CMV-treated group $(\mathrm{P}<0.05$; Fig. 7A). HSV-IGF-1 treatment also increased the expression ratios of pPI3K/PI3K and pAkt/Akt (HSV-IGF-1 vs. HSV-pNX-CMV, $\mathrm{P}<0.05$; Fig. 7B). However, HSV-siRNA-IGF-1 treatment reduced the number and extension of DRG neurons and decreased the pPI3K/PI3K and pAkt/Akt ratios, compared

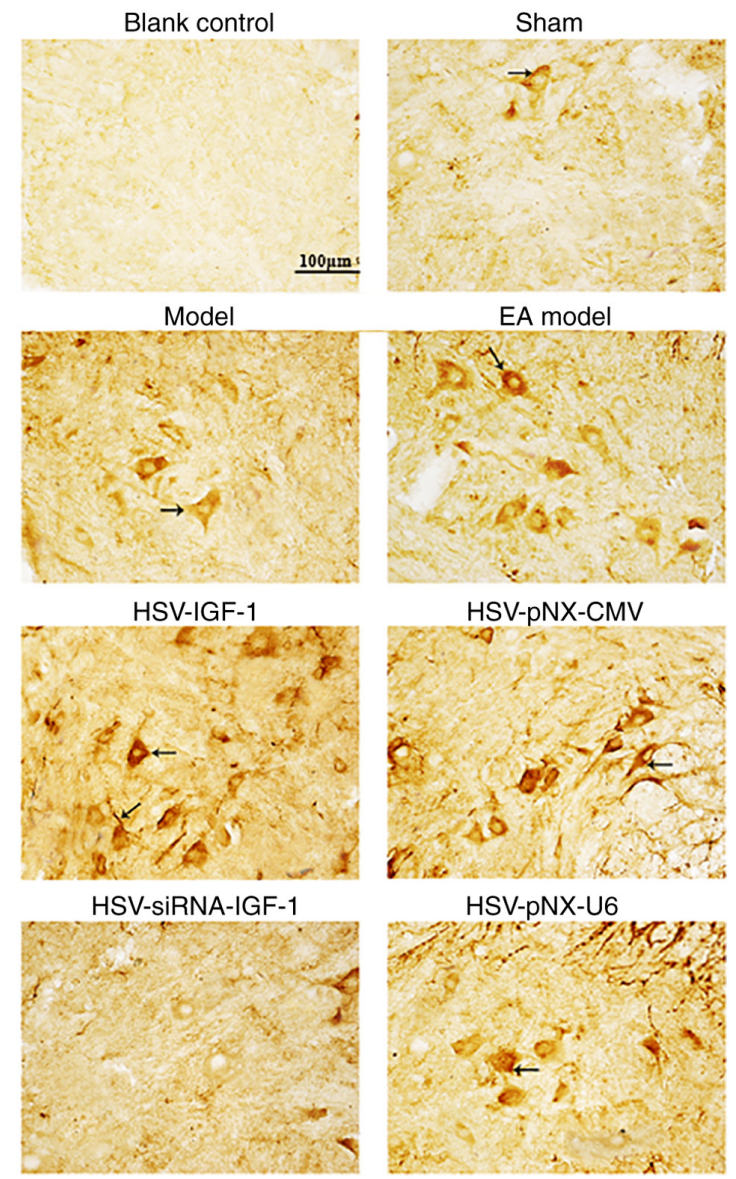

Figure 5. GAP-43-positive staining in different groups. Arrows indicate the representative files of GAP-43 stained files. Phosphate-buffered saline was substituted for the anti-GAP-43 antibody in the blank control. Magnification $\mathrm{x} 400$. Sham, sham-operated; Model, bilateral dorsal root ganglionectomy injury; EA Model, electro-acupuncture treatment post-injury; GAP-43, growth-associated protein 43; IGF-1, insulin-like growth factor 1; siRNA, small interfering RNA.

with those in the matched control group $(\mathrm{P}<0.05$; Fig. 7A and $\mathrm{B})$. In addition, HSV-IGF-1 induced DRG neuron growth, and the activation of PI3K/Akt was inhibited by pre-treatment with LY294002. Co-treatment with LY294002 in the HSV-IGF-1-transfected DRG neurons was associated with decreased DRG neuron numbers and extension, downregulation of the expression of pPI3K and pAkt, and decreased $\mathrm{pPI} 3 \mathrm{~K} / \mathrm{PI} 3 \mathrm{~K}$ and pAkt/Akt ratios (Fig. 8A and B). Consistent with these results, Akt siRNA induced a decrease in the phosphorylation of PI3K/Akt and the pPI3K/PI3K and pAkt/Akt ratios, and reduced the number and extension of DRG neurons, compared with those in cells co-transfected with HSV-IGF-1 and control scrambled siRNA ( $\mathrm{P}<0.05$; Fig. $8 \mathrm{~A}$ and $\mathrm{B})$.

\section{Discussion}

Previous studies have demonstrated that improvements in spinal neuroplasticity induced by EA therapy following SCI are associated with alterations in the expression of NTFs. Our previous study demonstrated that EA induced an increase in the expression of IGF-1 in the spared L6 DRG and associated dorsal horns of cats subjected to adjacent dorsal root 

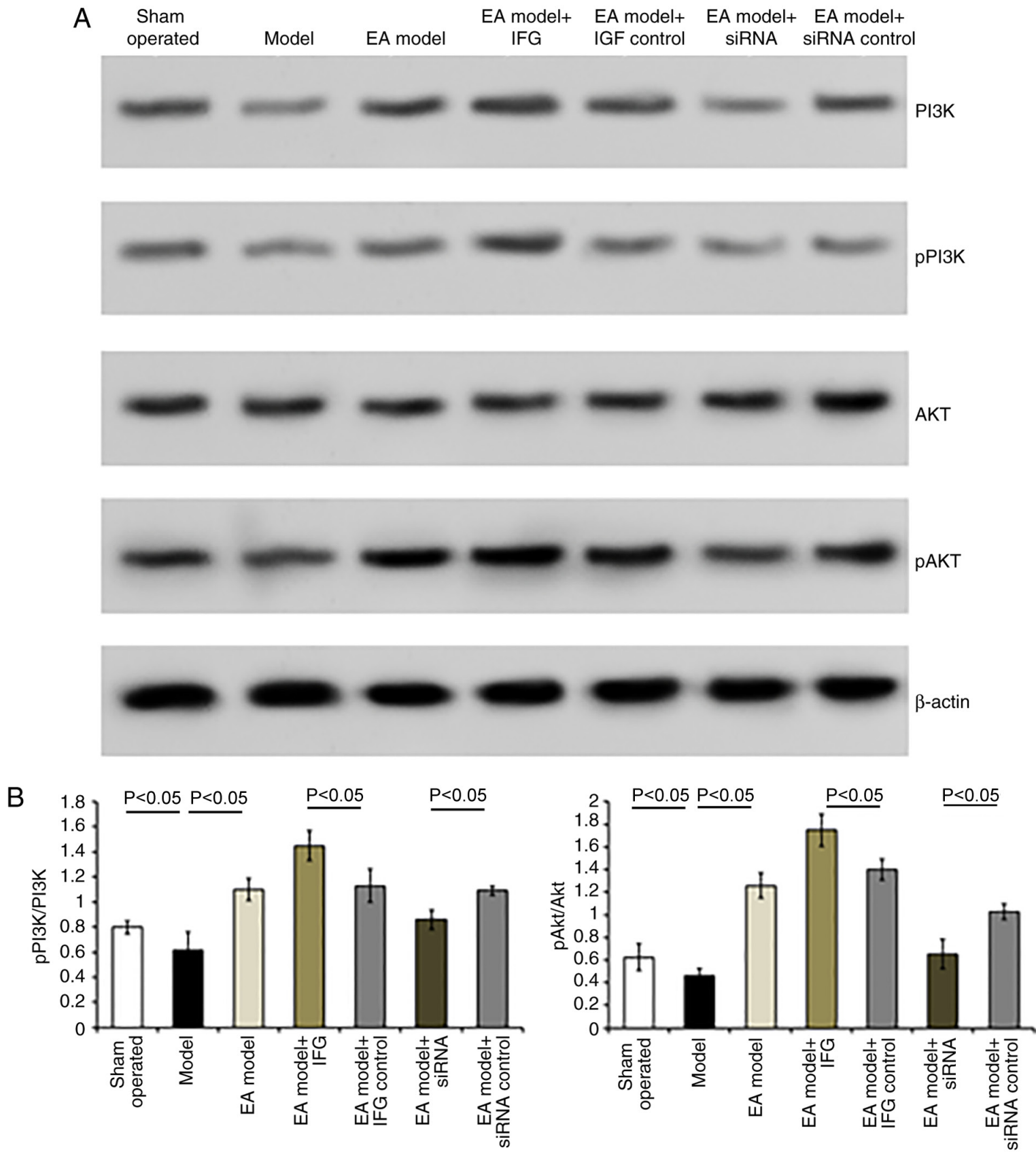

Figure 6. Detection of the expression of PI3K, pPI3K, Akt and pAkt in the different groups. (A) Representative blots of PI3K, pPI3K, Akt and pAkt proteins detected by western blot analysis. (B) Ratios of pPI3K/PI3K and pAkt/Akt. Values are plotted as the mean \pm standard deviation (n=7). Sham, sham-operated; Model, bilateral dorsal root ganglionectomy injury; EA Model, electro-acupuncture treatment post-injury; siRNA, small interfering RNA; PI3K, phosphatidylinositol 3-kinase; pPI3K, phosphorylated PI3K; pAkt, phosphorylated Akt.

ganglionectomies (26). However, the underlying mechanisms were unclear.

The results of the present study demonstrated that EA-induced repair and neuroplasticity following adjacent dorsal root ganglionectomies in rats were associated with IGF-1 via PI3K/Akt signaling pathway activation. Bilateral dorsal root ganglionectomies in rats reduced the expression of IGF-1 in the spared L5 DRG and impaired motor and sensory function. EA treatment partially rescued the expression levels of IGF-1, promoted the rehabilitation of locomotor function (detected using the BBB scale), remitted neuropathic pain (MWT and TWL test), increased CGRP- and GAP-43 immunopositivity in the L4-L5 spinal cord, and upregulated the $\mathrm{pPI} 3 \mathrm{~K} / \mathrm{PI} 3 \mathrm{~K}$ and pAkt/Akt ratios in the spared L5 DRG of the injured rats. The overexpression of IGF-1 by HSV-IGF-1 injection enhanced the effects induced by EA treatment. By contrast, interference of the expression of IGF-1 using targeted siRNA sequences neutralized the EA-induced effects in de-afferentated rats.

CGRP and GAP-43 serve key roles in regenerative neurite growth following spinal lesions (9). As a well-known marker for sensory axons transmitting pain sensations, the increased expression of CGRP is associated with nerve regeneration following lesion (44). GAP-43, another neuronal marker, is associated with nerve growth. It is a major component of the motile 'growth cones' that form the tips of elongating axons. 
A

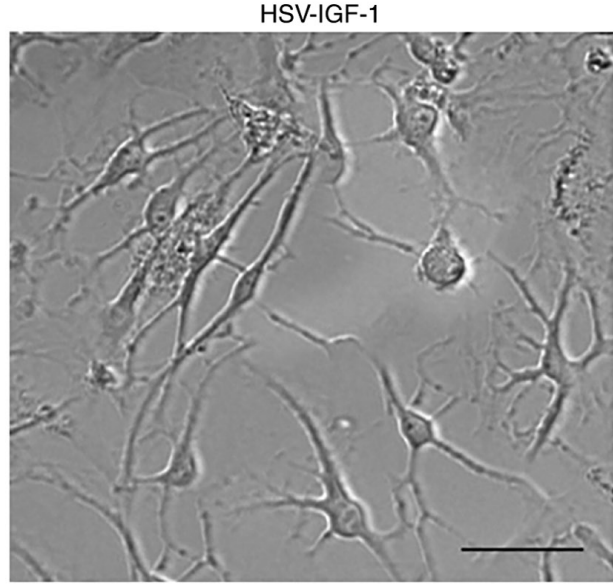

HSV-siRNA-IGF-1

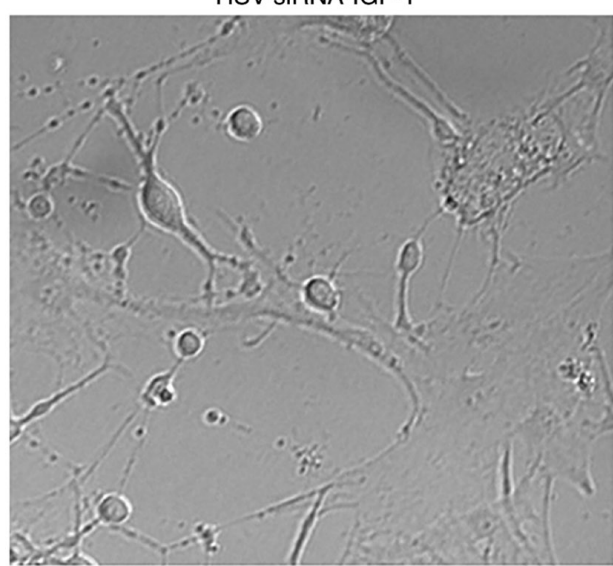

B
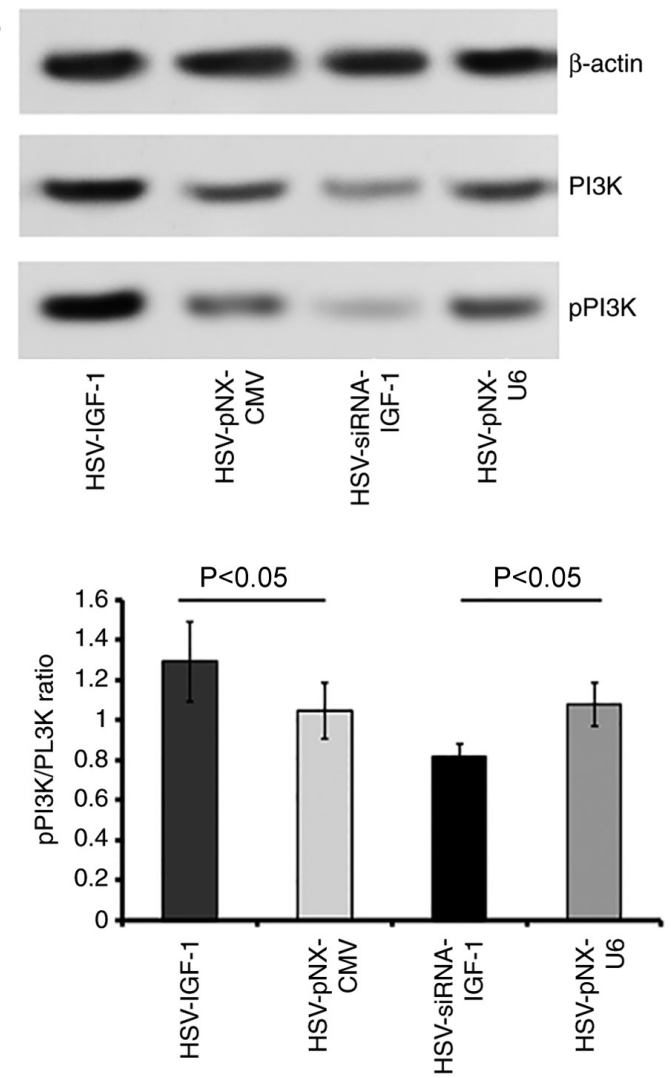

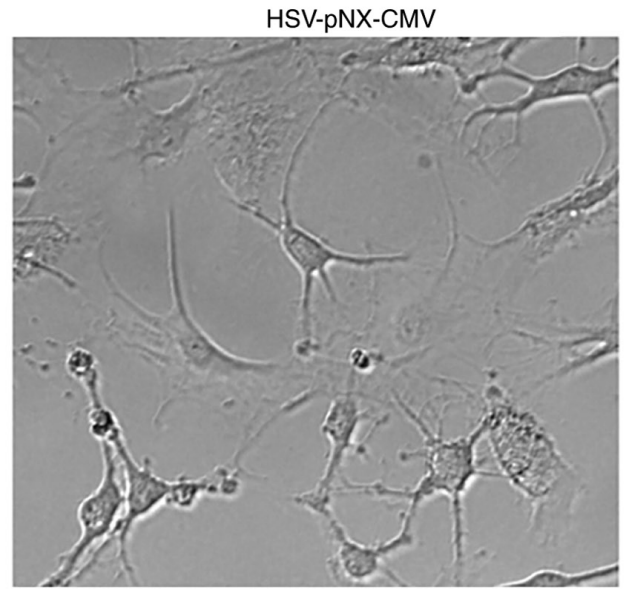

HSV-pNX-U6
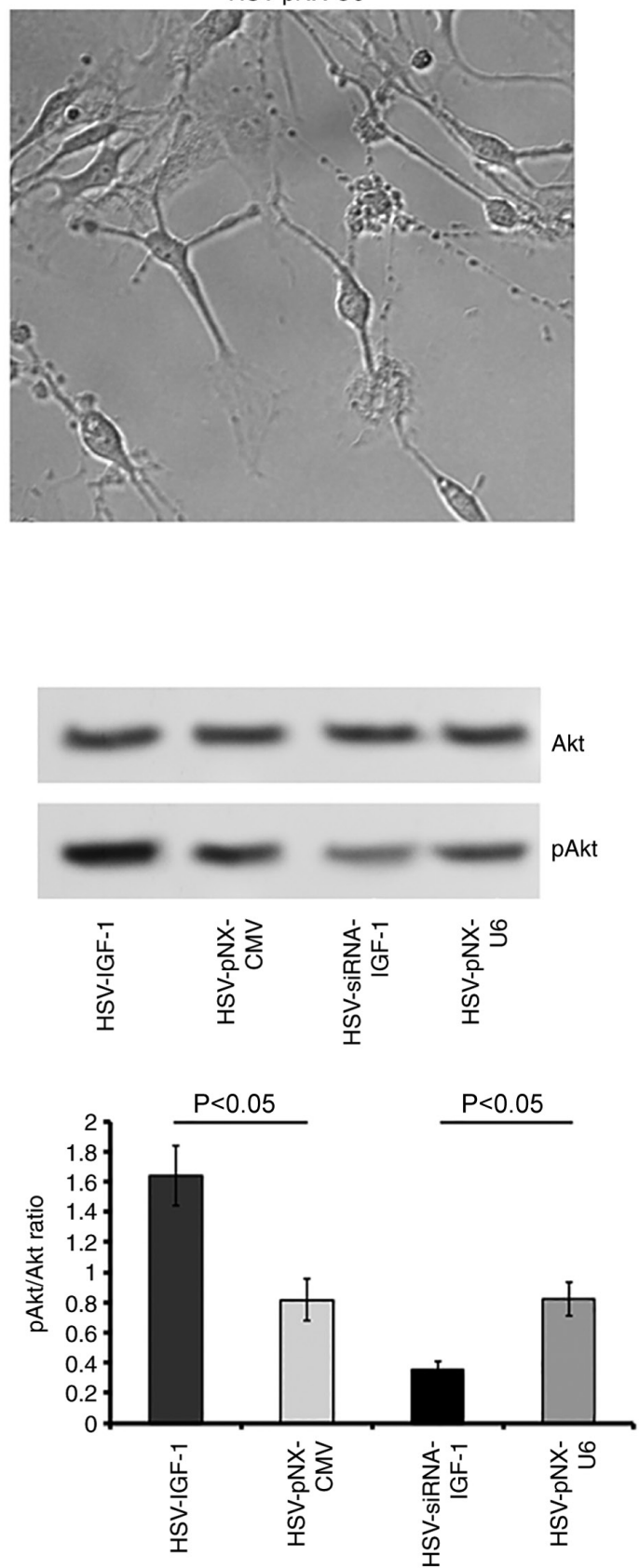

Figure 7. Effects of IGF-1 on cultured DRG neurons. (A) Morphological changes of cultured DRG neurons following treatment with artificial HSV-IGF-1, HSV-siRNA-IGF-1, HSV-pNX-CMV or HSV-pNX-U6 (magnification, x200). (B) Expression levels of PI3K and pPI3K, and the ratio of pAkt/Akt were evaluated by western blot analysis. Values are plotted as the mean \pm standard deviation ( $\mathrm{n}=5$ ). IGF-1, insulin-like growth factor 1 ; DRG, dorsal root ganglia; siRNA, small interfering RNA; PI3K, phosphatidylinositol 3-kinase; pPI3K, phosphorylated PI3K; pAkt, phosphorylated Akt. 
A

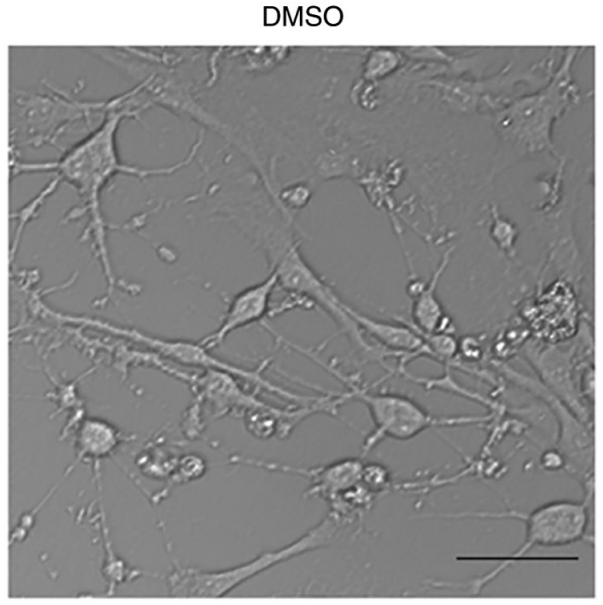

Scrambled siRNA

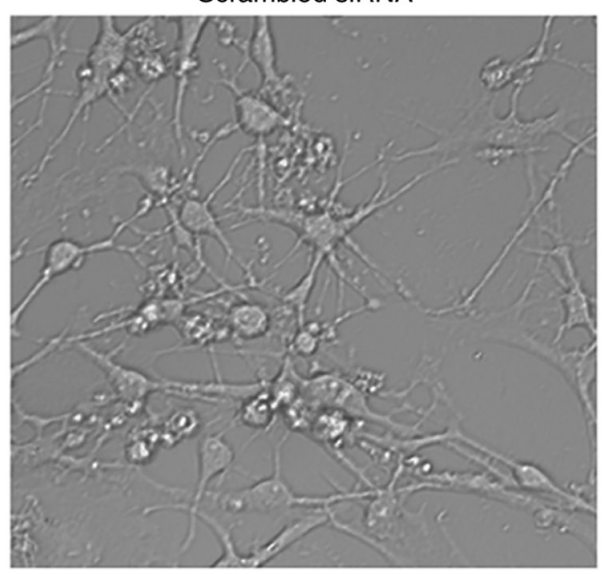

LY294002

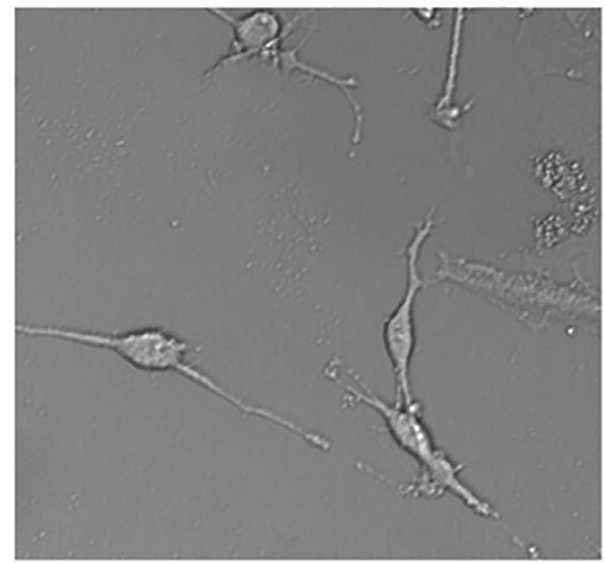

Akt siRNA

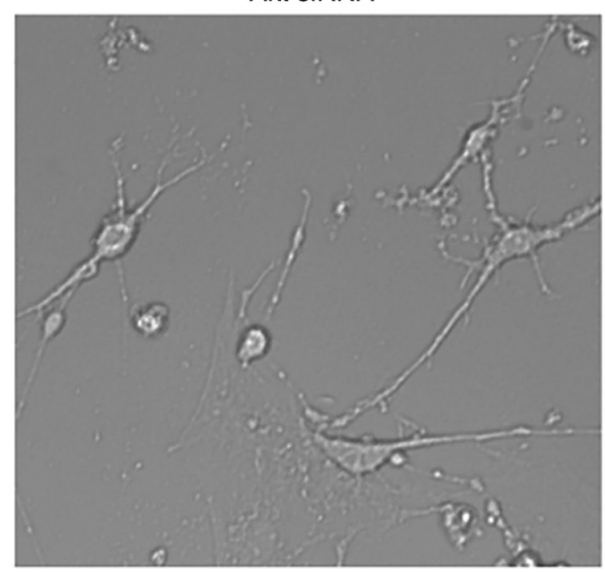

B
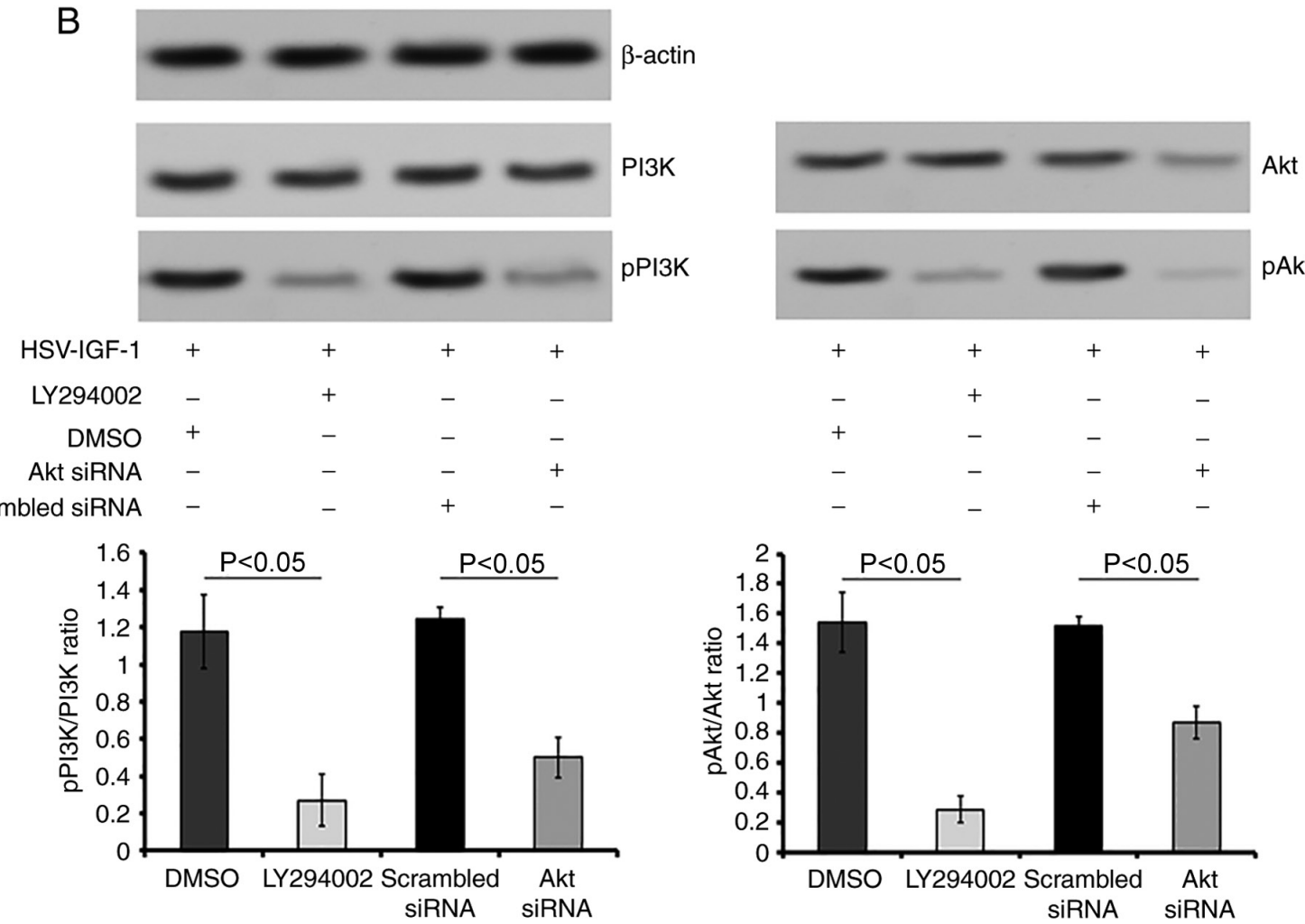

Figure 8. Roles of PI3K/Akt in IGF-1 induced neuroprotection in vitro. (A) Morphological changes of cultured DRG neurons following treatment with DMSO (control), LY294002, scrambled siRNA (control) and Akt siRNA in cultured DRG neurons transfected with HSV-IGF-1 (magnification, x200). (B) Representative blots of PI3K, pPI3K, Akt and pAkt proteins detected by western blot analysis. The ratios of pPI3K/PI3K and pAkt/Akt are also shown. Values are plotted as the mean \pm standard deviation $(\mathrm{n}=5)$. IGF-1, insulin-like growth factor 1; DRG, dorsal root ganglia; siRNA, small interfering RNA; PI3K, phosphatidylinositol 3-kinase; pPI3K, phosphorylated PI3K; pAkt, phosphorylated Akt; DMSO, dimethyl sulfoxide. 
Increases in the number of GAP-43-IRs indicate the regrowth of cones and synaptic formation in the spinal cord following injury (9). EA has been demonstrated to promote the recovery of sensory or motor functions in patients with SCI (5) and increase neuroplasticity in de-afferentated spinal cords $(6,7)$. Additional reports have shown that EA-induced increases in the expression of IGF-1 in the spared L6 DRG and associated dorsal horns may be associated with the intraspinal sprouting of DRG neurons in de-afferentated cats subjected to adjacent dorsal root ganglionectomies (26). Therefore, the enhanced CGRP- and GAP-43-IRs observed in the present study suggests that nerve regeneration, cone regrowth and synaptic formation were promoted in the de-afferentated spinal cord following EA treatment, and this may be used to reconstruct local circuitry for further functional recovery $(9,45)$. The results of the present study also demonstrated that the overexpression of IGF-1 promoted EA-induced neuroplasticity and that IGF-1 siRNA treatment weakened this effect. In addition, EA exerted a protective effect on de-afferentated spinal cords by stimulating the expression of IGF-1.

Following these observations, the present study investigated whether the EA-induced neuroplasticity associated with IGF-1 was mediated by PI3K/Akt activation. IGF-1 serves a neuroprotective and proliferative function in the CNS during development and following injury (15-17). A previous study revealed that IGF-1 receptor (IGF-1R)-knockout mice have reduced brain size, CNS hypomyelination, and loss of hippocampal granule cells and striatal parvalbumin-containing neurons (16). By contrast, transgenic mice overexpressing IGF-1 showed anti-apoptotic effects in neurons during early postnatal development of the cerebral cortex, which was associated with a persistent increase in the total number of neurons in the adult animal (15). IGF-1 has also been demonstrated to induce neuroprotection and neuroplasticity in de-afferentated spinal cords of cats (26). Initially, IGF-1 exerts its biological functions by binding to the specific receptor, IGF-1R. During normal insulin signaling, internalized IGF-1 binds to IGF-1R, which leads to the phosphorylation of IGF-1R and subsequent initiation of the downstream substrate, PI3K/Akt. Activated Akt phosphorylates glycogen synthase kinase-3 $\beta$ (GSK3 $\beta$ ) at Ser-9, leading to GSK3 $\beta$ inhibition and the prevention of pathogenic neuronal death. By contrast, the inhibition of Akt activates GSK $3 \beta$ and then stimulates aberrant tau phosphorylation, leading to neuronal death in certain diseases (46). In addition, the PI3K/Akt signaling pathway is involved in anti-apoptotic mechanisms, which influence the balance of anti- and pro-apoptotic proteins $(47,48)$. The Akt serine/threonine kinase is known to promote neuronal survival and serves as a key mediator of several aspects of neurite outgrowth, including elongation, branching and caliber (49). EA has been demonstrated to inhibit neuronal apoptosis in the dorsal root de-afferentated cat spinal cords, by regulating the expression of apoptosis-related proteins, including B-cell lymphoma 2 (Bcl-2) and Bcl-2-associated X protein (50).

The results of the present study revealed that EA treatment increased the expression of PI3K and the pPI3K/PI3K and $\mathrm{pAkt} / \mathrm{Akt}$ ratios, and this was enhanced by the upregulation of IGF-1and weakened following IGF-1 knockdown in the rat model. In cultured DRGs, the upregulation of IGF-1 induced DRG neuron outgrowth, increased the expression of pPI3K and pAkt, and increased the pAkt/Akt ratio. The downregulation of IGF-1 impeded DRG neuron extension, decreased the expression of PI3K, pPI3K and pAkt, and decreased the pAkt/Akt ratio. Furthermore, the inhibition of PI3K or Akt neutralized IGF-1 overexpression-induced neuroprotection in the cultured DRG neurons. Therefore, these results suggest that activation of the IGF-1/PI3K/Akt signaling pathway may be correlated with the EA-induced neuronal survival following de-afferentated SCI and may occur via activation of the IGF-1/PI3K/Akt signaling pathway.

As the regulation of neurite outgrowth is crucial for developing therapies that promote axon regeneration following nerve injury (49), IGF-1 is promising for the development of treatments for patients suffering with SCI.

In conclusion, the present study revealed the following regarding EA treatment: i) EA partially rescued IGF-1 levels and improved locomotor and sensory function; ii) EA enhanced CGRP- and GAP-43-IRs in the L4-L5 spinal cord; and iii) EA upregulated the ratios of $\mathrm{pPI} 3 \mathrm{~K} / \mathrm{PI} 3 \mathrm{~K}$ and $\mathrm{pAkt} / \mathrm{Akt}$ in the spared L5 DRG of rats that underwent bilateral dorsal root ganglionectomies. The overexpression of IGF-1 induced by the injection of HSV-IGF-1 into the spared L5 DRG reinforced the aforementioned observations regarding EA-induced neuroprotection. By contrast, downregulation of the endogenous expression of IGF-1 using specific siRNA IGF-1 target sequences neutralized these EA-induced effects. IGF-1 treatment in cultured DRGs led to neurite outgrowth and increased expression of $\mathrm{pPI} 3 \mathrm{~K} / \mathrm{PI} 3 \mathrm{~K}$ and pAkt/Akt. The results revealed the crucial role of IGF-1 in EA-induced neuroplasticity via the activation of PI3K/Akt following adjacent dorsal root ganglionectomies in rats.

\section{Acknowledgements}

Not applicable.

\section{Funding}

The present study was supported by Special Fund of the Applied Basic Research Programs of Yunnan Province associated with Kunming Medical University in China (grant no. 2015FB001), the Medical Reserve Talents Cultivation Project of the Health and Family Planning Commission of Yunnan province (grant no. H-2017026), the Foundation of Science and Technology innovative team building of Kunming Medical University (grant no. CXTD201807), the National Natural Science Foundation of China (grant nos. 81560238 and 81502377) and Yunnan Applied Basic Research Projects in China (grant nos. 2016FB139 and 2016FB123).

\section{Availability of data and materials}

The datasets used and analyzed in the present study are available from the corresponding author on reasonable request.

\section{Authors' contributions}

TH and YBX contributed to the conception and design of the study. YBX wrote and critically revised the manuscript. PD, MNL and BC established the injured model and administered 
the EA treatment. JT, MNL, RM, YXT, SSL and TH prepared the herpes simplex virus construction, injected the HSV vectors into the animals and performed the cell transfection. MNL, YXT, SSL and YBX determined the Basso, Beattie, Bresnahan scores rating, and performed the mechanical withdrawal threshold and thermal withdrawal latency evaluation. TH, MNL, BC, RM and YBX performed the reverse transcription-quantitative polymerase chain reactions, western blotting and immunohistochemical analyses. All authors read and approved the final manuscript.

\section{Ethics approval and consent to participate}

Animal use and care were in accordance with the animal care guidelines, which conformed to the Guide for the Care and Use of Laboratory Animals published by the US National Institutes of Health (publication no. 85-23; revised 1996) and the Care and Use Guidelines of Experimental Animals established by the Research Ethics Committee of Kunming University of China (permit no. kmu-eac-2016047).

\section{Patient consent for publication}

Not applicable.

\section{Competing interests}

The authors declare that they have no competing interests.

\section{References}

1. Morgan JI and Curran T: Immediate-early gene: Ten years on. Trends Neurosci 18: 66-67, 1995.

2. Sanner CA, Murray M and Goldberger ME: Removal of dorsal root afferents prevents retrograde death of axotomized Clarke's nucleus neurons in the cat. Exp Neurol 123: 81-90, 1993.

3. Zhang B, Goldberger ME and Murray M: Proliferation of SP- and 5HT-containing terminals in lamina II of rat spinal cord following dorsal rhizotomy: Quantitative EM-immunocytochemical studies. Exp Neurol 123: 51-63, 1993.

4. Zhang B, Goldberger ME, Wu LF and Murray M: Plasticity of complex terminals in lamina II in partially deafferented spinal cord: The cat spared root preparation. Exp Neurol 132: 186-193, 1995.

5. Wong AM, Leong CP, Su TY, Yu SW, Tsai WC and Chen CP. Clinical trial of acupuncture for patients with spinal cord injuries. Am J Phys Med Rehabil 82: 21-27, 2003.

6. Liu F, Sun WW, Wang Y, Hu LQ, Dai P, Tian CF and Wang TH: Effects of electro-acupuncture on NT-4 expression in spinal dorsal cats subjected to adjacent dorsal root ganglionectomy. Neurosci Lett 450: 158-162, 2009.

7. Wang XY, Li XL, Hong SQ, Xi-Yang YB and Wang TH: Electroacupuncture induced spinal plasticity is linked to multiple gene expressions in dorsal root deafferented rats. J Mol Neurosci 37: 97-110, 2009.

8. Zhou HL, Zhang LS, Kang Y, Zhang W and Wang TH: Effects of electro-acupuncture on CNTF expression in spared dorsal root ganglion and the associated spinal lamina II and nucleus dorsalis following adjacent dorsal root ganglionectomies in cats. Neuropeptides 42: 95-106, 2008.

9. Wang X, Ju S, Chen S, Gao W, Ding J, Wang G, Cao H, Tian H and Li X: Effect of electro-acupuncture on neuroplasticity of spinal cord-transected rats. Med Sci Monit 23: 4241-4251, 2017.

10. Sun WW, Zhao W and Wang TH: Effects of Electro-acupuncture on PDGF expression in spared dorsal root ganglion and associated dorsal horn subjected to partial dorsal root ganglionectomy in cats. Neurochem Res 33: 437-443, 2008.

11. Xu DU: Effect of electroacupuncture on brain derivd neurotrophic factor and nerve function of spinal cord injury rats. J Clin Acupuncture Moxibustion 2: 46-48, 2009 (In Chinese).
12. Cowansage KK, LeDoux JE and Monfils MH: Brain-derived neurotrophic factor: A dynamic gatekeeper of neural plasticity. Curr Mol Pharmacol 3: 12-29, 2010.

13. Russo VC, Schütt BS, Andaloro E, Ymer SI, Hoeflich A, Ranke MB, Bach LA and Werther GA: Insulin-like growth factor binding protein-2 binding to extracellular matrix plays a critical role in neuroblastoma cell proliferation, migration, and invasion. Endocrinology 146: 4445-4455, 2005.

14. Galvin J, Eyermann C and Colognato H: Dystroglycan modulates the ability of insulin-like growth factor-1 to promote oligodendrocyte differentiation. J Neurosci Res 88: 3295-3307, 2010.

15. Hodge RD, D'Ercole AJ and O'Kusky JR: Insulin-like growth factor-I (IGF-I) inhibits neuronal apoptosis in the developing cerebral cortex in vivo. Int J Dev Neurosci 25: 233-241, 2007.

16. Beck KD, Powell-Braxton L, Widmer HR, Valverde J and Hefti F: Igf1 gene disruption results in reduced brain size, CNS hypomyelination, and loss of hippocampal granule and striatal parvalbumin-containing neurons. Neuron 14: 717-730, 1995.

17. Hung KS, Tsai SH, Lee TC, Lin JW, Chang CK and Chiu WT: Gene transfer of insulin-like growth factor-I providing neuroprotection after spinal cord injury in rats. J Neurosurg Spine 6: 35-46, 2007.

18. Jung SY, Kim DY, Yune TY, Shin DH, Baek SB and Kim CJ: Treadmill exercise reduces spinal cord injury-induced apoptosis by activating the PI3K/Akt pathway in rats. Exp Ther Med 7: 587-593, 2014.

19. Craft S, Baker LD, Montine TJ, Minoshima S, Watson GS, Claxton A, Arbuckle M, Callaghan M, Tsai E, Plymate SR, et al: Intranasal insulin therapy for Alzheimer disease and amnestic mild cognitive impairment: A pilot clinical trial. Arch Neurol 69: 29-38, 2012.

20. Talbot K, Wang HY, Kazi H, Han LY, Bakshi KP, Stucky A, Fuino RL, Kawaguchi KR, Samoyedny AJ, Wilson RS, et al: Demonstrated brain insulin resistance in Alzheimer's disease patients is associated with IGF-1 resistance, IRS-1 dysregulation, and cognitive decline. J Clin Invest 122: 1316-1338, 2012.

21. Hölscher C: Insulin, incretins and other growth factors as potential novel treatments for Alzheimer's and Parkinson's diseases. Biochem Soc Trans 42: 593-599, 2014.

22. Morris JK, Zhang H, Gupte AA, Bomhoff GL, Stanford JA and Geiger PC: Measures of striatal insulin resistance in a 6-hydroxydopamine model of Parkinson's disease. Brain Res 1240: 185-195, 2008.

23. Bosco D, Plastino M, Cristiano D, Colica C, Ermio C, De Bartolo M, Mungari P, Fonte G, Consoli D, Consoli A and Fava A: Dementia is associated with insulin resistance in patients with Parkinson's disease. J Neurol Sci 315: 39-43, 2012.

24. Ashraghi MR, Pagano G, Polychronis S, Niccolini F and Politis M: Parkinson's disease, diabetes and cognitive impairment. Recent Pat Endocr Metab Immune Drug Discov 10: 11-21, 2016.

25. Pang Y, Lin S, Wright C, Shen J, Carter K, Bhatt A and Fan LW: Intranasal insulin protects against substantianigra dopaminergic neuronal loss and alleviates motor deficits induced by 6-OHDA in rats. Neuroscience 318: 157-165, 2016.

26. Dai P, Wang ZJ, Sun WW, Pang JX, You C and Wang TH: Effects of electro-acupuncture on IGF-I expression in spared dorsal root ganglia and associated spinal dorsal horn in cats subjected to adjacent dorsal root ganglionectomies. Neurochem Res 34: 1993-1998, 2009.

27. Fischer G, Kostic S, Nakai H, Park F, Sapunar D, Yu H and Hogan Q: Direct injection into the dorsal root ganglion: Technical, behavioral, and histological observations. J Neurosci Methods 199: 43-55, 2011.

28. Liu F, Zou Y, Liu S, Liu J and Wang T: Electro-acupuncture treatment improves neurological function associated with downregulation of PDGF and inhibition of astrogliosis in rats with spinal cord transection. J Mol Neurosci 51: 629-635, 2013.

29. Basso DM, Beattie MS and Bresnahan JC: A sensitive and reliable locomotor rating scale for open field testing in rats. J Neurotrauma 12: 1-21, 1995.

30. Vanderah TW, Gardell LR, Burgess SE, Ibrahim M, Dogrul A, Zhong CM, Zhang ET, Malan TP Jr, Ossipov MH, Lai J and Porreca F: Dynorphin promotes abnormal pain and spinal opioid antinociceptive tolerance. J Neurosci 20: 7074-7079, 2000.

31. Chaplan SR, Bach FW, Pogrel JW, Chung JM and Yaksh TL: Quantitative assessment of tactile allodynia in the rat paw. J Neurosci Methods 53: 55-63, 1994.

32. Liu FY, Sun YN, Wang FT, Li Q, Su L, Zhao ZF, Meng XL, Zhao H, Wu X, Sun Q, et al: Activation of satellite glial cells in lumbar dorsal root ganglia contributes to neuropathic pain after spinal nerve ligation. Brain Res 1427: 65-77, 2012. 
33. Deuis JR, Dvorakova LS and Vetter I: Methods used to evaluate pain behaviors in rodents. Front Mol Neurosci 10: 284, 2017.

34. Ding Y, Yao P, Hong T, Han Z, Zhao B, Chen W and Zhou G: Early hyperbaric oxygen effects on neuropathic pain and nitric oxide synthase isoforms in CCI rats. Oncotarget 9: 7513-7521, 2018.

35. Dixon WJ: Efficient analysis of experimental observations. Annu Rev Pharmacol Toxicol 20: 441-462, 1980.

36. Hargreaves K, Dubner R, Brown F, Flores C and Joris J: A new and sensitive method for measuring thermal nociception in cutaneous hyperalgesia. Pain 32: 77-88, 1988.

37. Fouillet A, Watson JF, Piekarz AD, Huang X, Li B, Priest B, Nisenbaum E, Sher E and Ursu D: Characterisation of Nav1.7 functional expression in rat dorsal root ganglia neurons by using an electrical field stimulation assay. Mol Pain 13: 17448069-17745179, 2017

38. Ren W, Liu Y, Wan S, Fei C, Wang W, Chen Y, Zhang Z, Wang T, Wang J, Zhou L, et al: BMP9 inhibits proliferation and metastasis of HER2-positive SK-BR-3 breast cancer cells through ERK1/2 and PI3K/AKT pathways. PLoS One 9: e96816, 2014.

39. Lu WD, Zuo Y, Xu Z and Zhang M: MiR-19a promotes epithelial-mesenchymal transition through PI3K/AKT pathway in gastric cancer. World J Gastroenterol 21: 4564-4573, 2015.

40. Hu T, Li YS, Chen B, Chang YF, Liu GC, Hong Y, Chen HL and Xiyang YB: Elevated glucose-6-phosphate dehydrogenase expression in the cervical cancer cases is associated with the cancerigenic event of high-risk human papillomaviruses. Exp Biol Med (Maywood) 240: 1287-1297, 2015.

41. Livak KJ and Schmittgen TD: Analysis of relative gene expression data using real-time quantitative PCR and the 2(-Delta Delta C(T)) method. Methods 25: 402-408, 2001.

42. Skup M, Dwornik A, Macias M, Sulejczak D, Wiater M and Czarkowska-Bauch J: Long-term locomotor training up-regulates TrkB (FL) receptor-like proteins, brain-derived neurotrophic factor, and neurotrophin 4 with different topographies of expression in oligodendroglia and neurons in the spinal cord. Exp Neurol 176: 289-307, 2002
43. Xiyang YB, Liu S, Liu J, Hao CG, Wang ZJ, Ni W, Wang XY and Wang TH: Roles of platelet-derived growth factor-B expression in the ventral horn and motor cortex in the spinal cord-hemisected rhesus monkey. J Neurotrauma 26: 275-287, 2009.

44. Jang JH, Nam TS, Paik KS and Leem JW: Involvement of peripherally released substance $\mathrm{P}$ and calcitonin gene-related peptide in mediating mechanical hyperalgesia in a traumatic neuropathy model of the rat. Neurosci Lett 360: 129-132, 2004.

45. Li WJ, Li SM, Ding Y, He B, Keegan J, Dong H, Ruan JW and Zeng YS: Electro-acupuncture upregulates CGRP expression after rat spinal cord transection. Neurochem Int 61: 1397-1403, 2012.

46. Yang L, Wang $H$, Liu L and $X i e ~ A$ : The role of insulin/IGF-1/PI3K/Akt/GSK3 $\beta$ signaling in parkinson's disease dementia. Front Neurosci 12: 73, 2018

47. Schorey JS and Cooper AM: Macrophage signaling upon mycobacterial infection: The MAP kinases lead the way. Cell Microbiol 5: 133-142, 2003

48. Chan G, Nogalski MT, Bentz GL, Smith MS, Parmater A and Yurochko AD: Pi3k-dependent upregulation of Mcl-1 by human cytomegalovirus is mediated by epidermal growth factor receptor and inhibits apoptosis in short-lived monocytes. J Immunol 184: 3213-3222, 2010.

49. Read DE and Gorman AM: Involvement of Akt in neurite outgrowth. Cell Mol Life Sci 66: 2975-2984, 2009.

50. Zhao W, Zhao Q, Liu J, Xu XY, Sun WW, Zhou X, Liu S and Wang TH: Electro-acupuncture reduces neuronal apoptosis linked to Bax and Bcl-2 expression in the spinal cords of cats subjected to partial dorsal root ganglionectomy. Neurochem Res 33: 2214-2221, 2008.

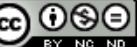

This work is licensed under a Creative Commons Attribution-NonCommercial-NoDerivatives 4.0 International (CC BY-NC-ND 4.0) License. 